\title{
On the different geographic characteristics of Free Trade Agreements and Customs Unions
}

\author{
James Lake* \\ Halis M. Yildiz ${ }^{\dagger}$ \\ Southern Methodist University \\ Ryerson University
}

June 19, 2014

\begin{abstract}
A striking phenomenon emerges from casual observation of the geographical characteristics of Preferential Trade Agreements (PTAs): while Customs Unions (CUs) are only intra-regional, Free Trade Agreements (FTAs) are both inter and intra-regional. A second striking phenomenon is that FTAs dramatically outnumber CUs. We present a farsighted dynamic model that endogenizes the choice of PTA type and rationalizes the first phenomenon via an FTA flexibility benefit: FTAs are more flexible than CUs because an FTA member is free to form further PTAs with non-members whereas a CU member must engage in further PTAs jointly with all existing members. Our model also suggests that greater distance between countries increases the prevalence of FTAs relative to CUs. Finally, the model relates geography and market size to the order of PTA formation.
\end{abstract}

JEL codes: C71, F12, F13

Keywords: Free Trade Agreement, Customs Union, flexibility, coordination, geography, networks, farsighted

\footnotetext{
*Southern Methodist University, Economics Department, 3300 Dyer Street, Dallas, TX 75275, USA. E-mail: jlake@smu.edu

${ }^{\dagger}$ Ryerson University, 350 Victoria Street, Toronto, ON M4K 1Y3, Canada. E-mail: hyildiz@economics.ryerson.ca
} 


\section{Introduction}

Many authors have documented the unabated proliferation of Preferential Trade Agreements (PTAs) that began in the early 1990s. Indeed, because of the inherently discriminatory nature of PTAs, this proliferation often motivates authors interest in the role that PTAs play in facilitating or hindering multilateral free trade. However, casual empiricism of PTA characteristics reveal two other striking observations that are often overlooked: i) unlike Free Trade Agreements (FTAs) which are both inter and intra-regional, Customs Unions (CUs) are only intra-regional and ii) FTAs are far more prevalent than CUs.

To be clear, the role of geography has always been intimately associated with PTA formation to the extent that "The terms "regional trade agreements" (RTAs) and "preferential trade agreements" (PTAs) are often used interchangeably in the literature" (WTO, 2011 p.58). Indeed, empirical evidence suggests that distance between countries plays a role in determining whether they have a PTA (e.g. Baier and Bergstrand (2004), Egger and Larch (2008), Chen and Joshi (2010) ). However, as one of the five stylized facts about PTAs, the WTO 2011, p.6) state that "PTA activity has transcended regional boundaries" and they go on to state that only $50 \%$ of all PTAs are regional. Thus, despite the intuitive appeal of "regionalism", surprisingly few papers have attempted to establish theoretical mechanisms underlying regionalism. ${ }^{1}$

More importantly, to the best of our knowledge, no paper has attempted to endogenously determine the choice of PTA type (i.e. CU or FTA) in a model where geographic asymmetry plays a role (by geographic asymmetry, we mean some countries are closer than others). In part, this open question stems from the fact that few papers explore the endogenous choice between CUs and FTAs. ${ }^{2}$ Our goal in this paper is to simultaneously address the issues of why FTAs and CUs differ in their geographical characteristics, whether geographic asymmetries can shed light on the prevalence of FTAs relative to CUs and the how the interaction between market size and geographic asymmetry affect the order in which FTAs form. ${ }^{3}$

We explore these issues in a three country dynamic model whose game theoretic setup is very similar to Seidmann (2009). Three features of our model are identical to Seidmann (2009): i) the possible agreements that can form in any given period, ii) at most one agree-

\footnotetext{
${ }^{1}$ One notable exception is Zissimos (2011). Another exception is a recent working paper by Soegaard (2013).

${ }^{2}$ The few exceptions include Riezman (1999), Melatos and Woodland (2007), Seidmann (2009), Facchini et al. (2012) Appelbaum and Melatos (2013), and Lake (2014).

${ }^{3}$ According to the WTO's RTA-IS database, http://rtais.wto.org/UI/PublicMaintainRTAHome. aspx accessed December 23 2013, 208 of the 225 PTAs in force and notified to the WTO under GATT Article XXIV are FTAs while the remaining 17 are CUs.
} 
ment can form in any given period, and iii) agreements formed in previous periods are binding. An additional similarity is that we assume proposer countries can propose agreements to other countries who, in turn, can reject or accept the proposed agreement. However, unlike Seidmann (2009) where the proposer country is stochastic, we assume a deterministic protocol where, similar to Aghion et al. (2007), the large country acts as a "leader country". Nevertheless, unlike Aghion et al. (2007), we allow the small countries to take the proposer role if i) they reject agreements proposed by the large country or ii) the large country chooses to make no proposal. In Section 3.1 we discuss reasons motivating our choice of this protocol including use of similar protocols in the earlier literature, its relationship to recent empirical work by Baier et al. (2014), its relationship to earlier versions of this paper where we attempted to fully endogenize which agreements would form in any given period, and avoiding difficulties associated with stochastic protocols given our results will emphasize intermediate values of the discount factor. In Section 5 , we demonstrate that our main results are robust to various alternative protocols including a small country being the "leader country".

For the underlying trade model, we add market size and geographic asymmetry to the popular competing exporters model of Bagwell and Staiger (1999). To focus on the role of geography, we assume trade between two "close" countries is not subject to transport costs while trade between either of the close countries and the third "far" country is subject to iceberg transport costs. Higher iceberg transport costs represent higher degrees of geographic asymmetry. When the degree of geographic asymmetry is low enough there is no meaningful distinction between intra and inter-regional agreements. However, with sufficient geographic asymmetry, we interpret an agreement involving the far country as inter-regional. In addition to geographic asymmetry, we believe market size asymmetry is also very important. Chen and Joshi (2010, p.244) find empirical evidence that, conditional on an FTA between a larger and a smaller country, the large country is more likely to form an FTA with an outsider country and become the hub. To focus on the role of market size asymmetry, we assume a large country and two smaller countries. In the main text of the paper, we assume the large country is the "far" country and the small countries are the "close" countries. Naturally there are contrary examples, but examples in line with Chen and Joshi (2010) and the context of our model are the sequences of FTAs involving the US, EU and EFTA as the large country and Israel, Jordan, Lebanon, Morocco, Australia and South Korea as smaller countries who are close to each other but far from the large country. ${ }^{4}$ We should emphasize that, as demonstrated in Section 5, we actually obtain stronger results when a small country

\footnotetext{
${ }^{4}$ The EU, EFTA and US (the large far country) formed sequential FTAs with Isreal and then Jordan (smaller close countries) before Jordan and Isreal concluded their own FTA. The same is true for the EU and EFTA with Lebanon and Morocco. Additionally, the US (large far country) formed sequential FTAs with Australia and Korea (smaller close countries) while negotiations are ongoing between Australia and Korea.
} 
is the "far" country.

A rich equilibrium structure emerges when we consider a "moderate degree" of market size asymmetry. Indeed, this equilibrium structure addresses the two key empirical observations of why CUs are intra-regional yet FTAs are inter and intra-regional and why FTAs are so prevalent relative to CUs.

With sufficient geographic asymmetry, and hence a meaningful distinction between inter and intra-regional agreements, we find that CUs are intra-regional yet FTAs are inter and intra-regional. This is our first main result. The key intuition is that, faced with the threat of being discriminated against by a small-small CU, the only type of PTA attractive enough to induce the large country's participation in liberalization is an FTA. How is this so? From a myopic perspective, $\mathrm{CU}$ formation is more attractive than FTA formation because it allows CU members to coordinate their external tariffs. However, from a dynamic perspective, FTA formation is more attractive than CU formation because of an FTA flexibility benefit: an individual FTA member can form subsequent PTAs with non-members unlike individual CU members who must engage in subsequent PTA formation jointly with all existing members. Rising market size and geographic asymmetry reduce the CU coordination benefit relative to the FTA flexibility benefit and the prospect of being discriminated against under a smallsmall CU. Indeed, this happens to the extent that FTA formation, but not CU formation, can induce the large country's participation in liberalization. When the FTA flexibility benefit is strong enough, a small country foregoes a permanent intra-regional CU with the other small country and engages in an inter-regional FTA with the large country because of valuable access to the large country market. A sequence of FTAs, inter and intra-regional, then lead to global free trade. Conversely, the large country opts out of PTA formation when the FTA flexibility benefit is weak enough and a permanent intra-regional CU between the small countries emerges.

The previous paragraph provided an explanation, primarily driven by the FTA flexibility benefit, for the observed phenomenon that CUs are only intra-regional yet FTAs are inter and intra-regional. In doing so, the model also provides an explanation for the observed prevalence of FTAs relative to CUs. However, this explanation operates only when geographic asymmetry is sufficiently large in which case there is a meaningful distinction between inter and intra-regional agreements. However, our model provides a different explanation linking rising geographic asymmetry with FTA rather than CU formation when geographic asymmetry is sufficiently small. Further, given the sufficiently small degree of geographic asymmetry, we interpret all agreements as intra-regional in this case. Thus, our model provides explanations linking rising geographic asymmetry to the prevalence of FTAs both when agreements are inter and intra-regional and when agreements are only intra-regional. 
Unlike the case where agreements are inter and intra-regional (i.e. geographic asymmetry is now sufficiently small), both CU formation and FTA formation can provide enough incentive for the large country to participation in liberalization when faced with the threat of being a CU outsider. Moreover, when the FTA flexibility benefit dominates the CU coordination benefit, a large and small country have divergent preferences over the type of PTA they should form: the large country prefers an FTA but the small country prefers a CU. Thus, to get its desired CU with the large country, the small country would like to credibly threaten the large country with being discriminated against under a small-small CU. However, this threat is only credible if the small country indeed prefers a small-small CU over an FTA with the large country. Moreover, the threat is not credible if a small-small CU does not expand to global free trade: the attractiveness to a small country of forming a small-small CU stems from eventually having access to the large country market under global free trade without having to endure the discrimination associated with being a spoke on a path of FTAs leading to global free trade. By reducing the large country's incentive to participate in expansion of a small-small CU to global free trade, rising geographic asymmetry mitigates the small country's ability to credibly threaten the large country. Thus, via its effect on a small country's ability to credibly threaten the large country into forming a small-large CU, rising geographic asymmetry helps explain the prevalence of FTAs even if all agreements are intra-regional.

Our paper clearly relates to the empirical determinants of PTA literature cited above, but it also bridges a gap between two distinct strands of the theoretical literature on PTA formation: (i) models where countries endogenously choose between FTAs and CUs but geography plays no role, and (ii) models where geography plays a role but countries do not endogenously choose between FTAs and CUs. ${ }^{5}$ In our model, geographically asymmetric countries endogenously choose between FTAs and CUs.

In the former strand of the literature, Riezman (1999) shows (in a setting with two small countries and one large country) that the threat of a CU between the small countries is necessary to induce the large country's participation in global free trade. In a similar setting, Melatos and Woodland (2007) show that consumer preference asymmetries reduce the CU coordination benefit to the extent that members may prefer FTAs over CUs. However, in both of these settings, and in contrast to our paper, FTAs never emerge in a unique equilibrium. Appelbaum and Melatos (2013) show how uncertainty over demand and marginal cost can affect the attractiveness of CUs relative to FTAs by affecting the benefit of exter-

\footnotetext{
${ }^{5}$ In addition, some papers have compared an "FTA formation game" with a "CU formation game" rather than endogenized the chioce between FTAs and CUs. Examples here include Furusawa and Konishi (2007) and Missios et al. (2014).
} 
nal tariff coordination. Facchini et al. (2012) show how PTAs emerge in equilibrium when income inequality is low with FTAs rather than CUs emerging when cross country production structures are sufficiently different. Unlike these static models, Lake (2014) develops a dynamic model and shows a necessary and sufficient condition for multiple FTAs to emerge in equilibrium is that the FTA flexibility benefit dominates the CU coordination benefit.

Seidmann (2009) also develops a dynamic model. In a three country dynamic bargaining model with transfers, he shows that PTAs can be valuable because of a "strategic positioning" motive: PTA members can affect their share of the global free trade pie by affecting the outside option of the PTA outsider. Because exploiting the strategic positioning motive requires direct expansion of the bilateral PTA to global free trade, CUs may be preferable to FTAs because CU expansion must immediately result in global free trade whereas FTA expansion can produce overlapping FTAs. Thus, while the flexibility of FTAs mitigates the strategic positioning motive for PTA formation in Seidmann (2009), it is a benefit in our framework. Additionally, there is no role for geography in Seidmann (2009).

A key modeling difference between our paper and Seidmann (2009) driving the different role of FTAs is the absence of transfers. Without transfers, countries payoffs come from the discounted value of one period payoffs along the equilibrium path. Thus, the CU coordination benefit arises because of the additional one period payoff between CU and FTA formation stemming from external tariff coordination whereas FTA formation is valuable because it allows a country to then become the hub and have sole preferential access to each spoke market. Additionally, even though global free trade maximizes world welfare (here and in Seidmann (2009) ), global free trade may not arise here given the absence of transfers (as in, e.g., Saggi et al. (2013)). Bagwell and Staiger (2010, p.50) argue that reality is "... positioned somewhere in between the extremes of negotiations over tariffs only and negotiations over tariffs and [transfers]...". ${ }^{6}$

Similar to the role of the FTA flexibility benefit in our model, Melatos and Dunn (2013) build a two period model illustrating that FTA formation between two non-autarkic countries may be more attractive than CU formation when they anticipate an autarkic third country will subsequently integrate themselves into world trade. In contrast, our setting is one where all countries participate in global trade in all periods. ${ }^{7}$

\footnotetext{
${ }^{6}$ There are papers allowing transfers between countries (e.g. Aghion et al. (2007), Ornelas (2008), and Bagwell and Staiger (2010)) while other papers assume away transfers (e.g. Riezman (1999), Furusawa and Konishi (2007), Melatos and Woodland (2007), Saggi and Yildiz (2010) and Facchini et al. (2012)). Bagwell and Staiger (2010, p.50) state that "While it is not standard for GATT/WTO trade negotiations to involve explicit transfers as part of the agreement, these negotiations do often involve more than just tariff reductions." and Furusawa and Konishi (2007, p.329) state "...feasible amounts of transfer are usually limited in practice.".

${ }^{7}$ While the setting in Melatos and Dunn (2013) has the spirit of WTO ascension after the 1995 inception
} 
In the strand of the literature not considering the endogenous choice between FTAs and CUs, Ludema (2002) builds a three country economic geography model. Global free trade is not attainable once any country is sufficiently far from the others. When there are two sufficiently close countries and one far country (similar to the geographic structure we consider), an FTA between the close countries emerges as the unique equilibrium. In a model of coalition formation where multiple equilibria emerge, Zissimos (2011) argues that regionalism, via larger trade volumes arising from lower transport costs, could stem from countries using proximity to coordinate on a unique equilibrium. Soegaard (2013) shows how greater product variety diminishes the incentive for regionalism and increases the scope for global free trade. Our paper differs from these three papers because we endogenize the choice between FTAs and CUs in addition to the role played by geography.

The paper proceeds as follows. Section 2 presents the underlying trade model which is a modified version of the Bagwell and Staiger (1999) competing exporters model. Section 3 presents the dynamic game. Section 4 explores the equilibrium path of agreements in the dynamic game and Section 5 discusses how our model is robust to alternative modeling choices. Finally, Section 6 concludes. Proofs are collected in Appendix B.

\section{Underlying trade model}

For our underlying trade model, we utilize an appropriately adapted version of the competing exporters framework developed by Bagwell and Staiger (1999). There are three countries: $i, j$, and $k$; three non-numeraire goods: $I, J$, and $K$; and a numeraire good $y$. Each country's market is served by two competing exporters and country $i$ is endowed with zero units of good $I$ and $e_{i}$ units of the other two goods. ${ }^{8}$

To this standard endowment structure, we add market size and geographic asymmetry. The demand for good $z$ in country $i$ is given by $q\left(p_{i}^{z}\right)=\alpha_{i}-p_{i}^{z}$ where $z=I, J$, or $K{ }^{9}$ Since each country possesses only two goods while it demands all three, country $i$ must import good $I$ in order to consume it and can import it from either trading partner. For example, country $i$ imports good $I$ from both countries $j$ and $k$ while it exports good $J$ to country $j$ and good $K$ to country $k$. Given linear demand, the intercept on the inverse demand curve is

of the WTO (e.g. Russia, China, Jordan or Vietnam) it should be noted that non-WTO members are generally not autarkic prior to WTO ascension and even form PTAs notified to the WTO under GATT Article XXIV (e.g. Russia).

${ }^{8}$ In addition, all countries have large enough endowments of the numeraire good $y$ to ensure balanced trade.

${ }^{9}$ As is well known, these demand functions can be derived from a utility function of the form $U\left(q^{z}\right)=$ $\sum_{z} u\left(q^{z}\right)+y$ where $q^{z}$ denotes consumption of good $z$ and $y$ denotes the numeraire good. 
interpreted as the market size parameter. As discussed in the introduction, we model market size asymmetry through two small countries $s_{1}$ and $s_{2}$ and one large country $l$. When there is no need to distinguish between $s_{1}$ and $s_{2}$ we merely denote a small country by $s$.

We model geographic asymmetry via traditional iceberg transport costs. Specifically, only a fraction $\tau_{i j}$ of a unit shipped from country $i$ actually arrives in country $j$. Thus, a lower $\tau_{i j}$ indicates higher transport costs and a greater degree of geographic asymmetry. To focus on the role of geography, we assume that trade is costless between the small countries $s_{1}$ and $s_{2}$ so that $\tau_{s_{1} s_{2}}=\tau_{s_{2} s_{1}}=1$. Thus, we call the small countries "close". Conversely, trade is costly between either of the close countries $s$ and the third "far" country $l: \tau_{s l}=\tau_{l s}=\tau \leq 1$. Later, we will interpret a bilateral PTA involving the large far country as "inter-regional" and a bilateral PTA involving the small close countries as an "intra-regional" agreement. However, when geographic asymmetry is low enough (i.e. $\tau$ large enough) we will interpret all bilateral PTAs as intra-regional even though there is some degree of geographic asymmetry.

Letting $t_{i j}$ be the tariff imposed by country $i$ on its imports of good $I$ from country $j$, and ruling out prohibitive tariffs, yields the following no-arbitrage conditions for good $I$ :

$$
p_{i}^{I}=\frac{p_{j}^{I}}{\tau_{i j}}+t_{i j}=\frac{p_{k}^{I}}{\tau_{i k}}+t_{i k} \text { where } i \neq j \neq k
$$

Let $m_{i}^{I}$ be country $i$ 's imports of good $I$. Since country $i$ has no endowment of good $I$, we have

$$
m_{i}^{I}=q\left(p_{i}^{I}\right)=\alpha_{i}-p_{i}^{I}
$$

Each country exports its endowment of a good minus its local consumption. Thus, export supply of country $j$ to country $i$ is $\left[e_{j}-\left(\alpha_{j}-p_{j}^{I}\right)\right]$. However, only a fraction $\tau$ of these exports arrive. Thus, the exports from country $j$ that arrive in country $i$ are

$$
x_{j}^{I}=\tau_{i j}\left[e_{j}-\left(\alpha_{j}-p_{j}^{I}\right)\right]
$$

Market clearing for good $I$ requires that country $i$ 's imports equal the total exports of the other two countries (denoted by $x^{I}$ ):

$$
m_{i}^{I}=x^{I}=\sum_{h \neq i} x_{h}^{I}
$$


Equations (1) through (4) imply that the equilibrium price of good $I$ in country $i$ equals:

$$
p_{i}^{I}=\frac{\alpha_{i}+\sum_{h \neq i}\left[\tau_{h i}\left(t_{i h} \tau_{h i}+\alpha_{h}-e_{h}\right)\right]}{1+\sum_{h \neq i} \tau_{h i}^{2}} .
$$

As is clear from (5), the price of good $I$ in country $i$ increases in the transportation cost (supply side effect), market size of all countries (demand side effect) and its tariffs. The effect of a country's tariff on its terms of trade is evident from equation (5): only a fraction of a given increase in either of its tariffs $\left(\tau_{h i}^{2} /\left(1+\sum_{h \neq i} \tau_{h i}^{2}\right)\right)$ is passed on to domestic consumers. Using these prices, the volume of trade is easily calculated:

$$
x_{j}^{I}=\tau_{j i} \frac{\left(e_{j}-\alpha_{j}+\tau_{j i}\left(\alpha_{i}-t_{i j}\right)\right)+\tau_{k i}^{2}\left(e_{j}-\alpha_{j}\right)-\tau_{j i} \tau_{k i}\left(e_{k}-\alpha_{k}\right)+\tau_{j i} \tau_{k i}^{2}\left(t_{i k}-t_{i j}\right)}{1+\sum_{h \neq i} \tau_{h i}^{2}}
$$

and thus

$$
m_{i}^{I}=x^{I}=\frac{\left.\sum_{h \neq i}\left[\tau_{h i}\left(e_{h}-\alpha_{h}\right)+\tau_{h i}^{2}\left(\alpha_{i}-t_{i h}\right)\right)\right]}{1+\sum_{h \neq i} \tau_{h i}^{2}} .
$$

From a welfare perspective, given the effective partial equilibrium nature of the model, it suffices to consider only protected goods and ignore the numeraire good. Thus, a country $i$ 's welfare is defined as the sum of consumer surplus, producer surplus, and tariff revenue over all such goods:

$$
W_{i}=\sum_{z} C S_{i}^{z}+\sum_{z} P S_{i}^{z}+T R_{i}
$$

Using equations (1) through (5) one can obtain the welfare of country $i$ as a function of endowment levels and tariffs. We next derive optimal tariffs under each regime. ${ }^{10}$ From hereon, we assume that the endowment of goods in each country is normalized to $1: e_{h}=1$, where $h=i, j, k$. To capture market size asymmetry, we set the market size of small countries to $\alpha_{s}=1$ and let $\alpha_{l}>1$.

\footnotetext{
${ }^{10}$ Calculations supporting the results reported in the rest of the paper are contained in the appendix.
} 


\subsection{Optimal Tariffs}

\subsubsection{No Agreements}

In the absence of any trade agreement, denoted $\varnothing$, each country $i$ chooses a non-discriminatory tariff (in accordance with GATT Article I) $t_{i}=t_{i j}=t_{i k}$ to maximize its welfare. ${ }^{11}$ As is well known (e.g. Feenstra (2004), Broda et al. (2008) and Missios et al. (2014)), country $i$ 's optimal tariff can be represented generally as:

$$
t_{i}(\varnothing)=x^{I} \frac{\partial p_{w}^{I}}{\partial x_{j}^{I}}
$$

where $p_{w}^{I}$ is the world price of good $I$. These optimal MFN tariffs of the small (close) countries $s$ and the large (far) country $l$ are easily calculated:

$$
t_{s}(\varnothing) \equiv \operatorname{Arg} \max W_{s}(\varnothing)=\frac{1-\tau\left(\alpha_{l}-1\right)+\tau^{2}}{\left(1+\tau^{2}\right)\left(3+\tau^{2}\right)}
$$

and

$$
t_{l}(\varnothing) \equiv \operatorname{Arg} \max W_{l}(\varnothing)=\frac{\alpha_{l}}{2\left(1+\tau^{2}\right)} .
$$

It is immediate from the above tariffs that as transportation costs rise (i.e. as $\tau$ falls), optimal tariffs rise: $\frac{\partial t_{l}(\varnothing)}{\partial \tau}<0$ and $\frac{\partial t_{s}(\varnothing)}{\partial \tau}<0$. To understand the intuition, suppose that countries are symmetric in market size and thus their import demands are identical. Note that the export supply is linear and goes through the origin in our model, and thus $x_{j}^{I} \frac{\partial p_{w}^{I}}{\partial x_{j}^{I}}$ reduces to $p_{w}^{I}$. As transportation costs rise ( $\tau$ falls), the export supply curve of a good traded between far apart countries becomes steeper (higher $\left.\frac{\partial p_{w}^{I}}{\partial x_{j}^{I}}\right)$ leading to a higher equilibrium world price $p_{w}^{I}$. Thus, using (8), optimal tariffs increase with transportation costs.

Moreover, as the market size of the large country rises, its own tariff rises while small countries impose lower tariffs: $\frac{\partial t_{l}(\varnothing)}{\partial \alpha_{l}}>0$ and $\frac{\partial t_{s}(\varnothing)}{\partial \alpha_{l}}<0$. To see this more clearly, suppose there exists no transportation cost: $\tau=1$. As $\alpha_{l}$ rises, the import demand of the large country shifts parallel to the right, leading to a larger equilibrium export volume into country l. Since the slope of the export supply curve stays unchanged, (8) shows the optimal tariff of the large country rises as $\alpha_{l}$ increases. On the other hand, the export supply of the large country shifts parallel to the left as $\alpha_{l}$ rises, leading to a smaller equilibrium volume of exports into small countries. Therefore, given a constant slope of the export supply curve, (8) shows the optimal tariff of a small country falls as $\alpha_{l}$ increases.

Given country $l$ has a larger market size and faces transport costs when importing from

\footnotetext{
${ }^{11}$ See Figure 1 in Section 3.1 for all possible networks of trade agreements and their notation.
} 
both trading partners, it imposes higher tariffs relative to the small countries:

$$
t_{l}(\varnothing)-t_{s}(\varnothing)=\frac{\alpha_{l}\left(\tau^{2}+2 \tau+3\right)-2\left(\tau^{2}+\tau+1\right)}{2\left(1+\tau^{2}\right)\left(3+\tau^{2}\right)}>0
$$

Note that, for sufficiently small $\tau$ and sufficiently large $\alpha_{l}$, exports from country $l$ can be negative. To exclude this possibility, we assume $\alpha_{l} \leq \bar{\alpha}_{l}^{x}(\tau)$ where $^{12}$

$$
\bar{\alpha}_{l}^{x}(\tau) \equiv 1+\frac{\tau\left(\tau^{2}+1\right)}{2 \tau^{2}+3}
$$

\subsubsection{Free Trade Agreements}

Under a single FTA between countries $i$ and $j$, denoted $g_{i j}$, they remove their tariffs on each other and impose their optimal external tariffs on the non-member country. Under the single FTA between $s_{1}$ and $l$, we have: ${ }^{13}$

$$
\begin{gathered}
t_{s_{1} s_{2}}\left(g_{s_{1} l}\right) \equiv \operatorname{Arg} \max W_{s}\left(g_{s_{1} l}\right)=\frac{\tau\left(3+\tau^{2}\right)\left(\alpha_{l}-1\right)+1}{\left(1+2 \tau^{2}\right)\left(2+\tau^{2}\right)+\left(1+\tau^{2}\right)} \\
t_{l_{s_{2}}}\left(g_{s_{1} l}\right) \equiv \operatorname{Arg} \max W_{l}\left(g_{s_{1} l}\right)=\frac{\alpha_{l}}{\left(1+2 \tau^{2}\right)^{2}+\left(1+\tau^{2}\right)}
\end{gathered}
$$

It is immediate that member tariffs rise with transportation costs (i.e. as $\tau$ falls). The effect is direct for $l$ : country $l$ faces a steeper export supply curve as $\tau$ falls and thus imposes higher tariffs as explained above in the No Agreements case. The effect is indirect for $s_{1}$ : higher transportation costs induce $s_{1}$ to reallocate imports from its FTA partner $l$ to the non-member $s_{2}$ and, in turn, raise its tariff on $s_{2}$. Since the former direct effect dominates the latter indirect effect, l's tariff rises faster than $s_{1}$ 's tariff as $\tau$ falls.

As $\alpha_{l}$ rises, both member countries impose higher tariffs. First, note that a higher $\alpha_{l}$ raises the import demand of country $l$ as under the No Agreement case. However, unlike the No Agreement case, most of the increased imports still come from its FTA partner which limits the increase of its external tariff. From the small member country's perspective, unlike the No Agreement case, it imposes higher tariffs as $\alpha_{l}$ rises. Indeed, given the absence of any limiting effect, the small member's tariff rises faster than the large member's tariff as $\alpha_{l}$ rises. To understand why the external tariff of $s_{1}$ rises, note that export supply of $l$ shrinks as its demand rises. Thus, $s_{1}$ relies more on imports from the non-member country which

\footnotetext{
${ }^{12}$ Later in the tariff complementarity discussion under an FTA between $s_{1}$ and $s_{2}$, we will argue that this condition does not bind.

${ }^{13}$ Since the non-member country is the sole importer of the good exported by the member countries, we have $t_{k}(\varnothing)=t_{k}\left(g_{i j}\right)$. In a "hub-spoke" network where $i$ is the hub (see Figure 11 we have $t_{k j}\left(g_{i}^{H}\right)=$ $t_{j k}\left(g_{i}^{H}\right)=t_{j k}\left(g_{i j}\right)$ for spoke countries $j$ and $k$. In contrast, since the hub has an FTA with both spokes, it practises free trade.
} 
raises its external tariff.

An implication of this discussion is that the small member imposes a higher tariff than the large member under an FTA when there is no transportation cost: $t_{s_{1} s_{2}}\left(g_{s_{1} l}\right)>t_{l s_{2}}\left(g_{s_{1} l}\right)$ when $\tau=1$. But, since the large member's tariff rises faster than the small member's tariff as $\tau$ falls, there exists a threshold $\underline{\tau}\left(\alpha_{l}\right)$ below which the large member imposes a higher tariff relative to the small member. It is also immediate from the above tariffs that formation of a bilateral FTA induces each member to lower its tariff on the non-member country relative to no agreement (i.e. the model exhibits tariff complementarity). ${ }^{14}$

Now consider the formation of a single FTA between two small countries. The members' optimal external tariffs are:

$$
t_{s_{1} l}\left(g_{s_{1} s_{2}}\right)=t_{s_{2} l}\left(g_{s_{1} s_{2}}\right) \equiv \operatorname{Arg} \max W_{s_{1}}\left(g_{s_{1} s_{2}}\right)=\frac{\left(4+\tau^{2}\right)\left(1-\alpha_{l}\right)+\tau}{\tau\left(3 \tau^{2}+8\right)}
$$

Note that when $\alpha_{l}$ and $\tau$ are sufficiently large, it is optimal for small countries to impose an import subsidy:

$$
t_{s l}\left(g_{s_{1} s_{2}}\right)=t_{s l}\left(g_{s_{1} s_{2}}\right)<0 \text { when } \alpha_{l}>\bar{\alpha}_{l}^{t}(\tau) \equiv 1+\frac{\tau}{4+\tau^{2}}
$$

To guarantee non-negative external tariffs, we assume that $\alpha_{l} \leq \bar{\alpha}_{l}^{t}(\tau)$ holds hereafter. It is worth noting that $\bar{\alpha}_{l}^{t}(\tau) \leq \bar{\alpha}_{l}^{x}(\tau)$ always holds and thus the previous condition avoiding negative exports is no longer binding.

A similar tariff discussion applies here as above. ${ }^{15}$ An important difference is that as $\alpha_{l}$ rises, small member countries have an incentive to reduce their external tariffs. As the demand of country $l$ rises, its export supply shifts to the left, reducing the volume of imports by member countries which are exports of the non-member. Thus, member countries lower their external tariffs relative to the No Agreement case.

\subsubsection{Customs Unions}

Under a single CU between countries $i$ and $j$, denoted $g_{i j}^{C U}$, they remove tariffs on each other and impose jointly optimal external tariffs by solving the following joint welfare maximization

\footnotetext{
${ }^{14}$ See Richardson (1993), Bagwell and Staiger (1999), and Saggi and Yildiz $(2009)$ for a detailed discussion of the tariff complementarity effect and Estevadeordal et al. (2008) for empirical evidence in its support. It is worth noting that tariff complementarity also arises in simple general equilibrium models of trade agreements such as Bond et al. (2004).

${ }^{15}$ It is easy to see that the tariff complementarity effect holds under $g_{s_{1} s_{2}}$ as well.
} 
problem: ${ }^{16,17}$

$$
\max _{t_{i}\left(g_{i j}^{C U}\right), t_{j}\left(g_{i j}^{C U}\right)} W_{i}\left(g_{i j}^{C U}\right)+W_{j}\left(g_{i j}^{C U}\right) \text { subject to } t_{i j}\left(g_{i j}^{C U}\right)=t_{j i}\left(g_{i j}^{C U}\right)=0 \text {. }
$$

CU members benefit from tariff coordination because they internalize the effect their external tariffs have each other's export surplus. ${ }^{18}$

Under a CU between $s_{1}$ and $l$ we have:

$$
t_{s_{1} s_{2}}\left(g_{s_{1} l}^{C U}\right)=\frac{\tau\left(\alpha_{l}-1\right)+1}{\left(2 \tau^{2}+3\right)} \text { and } t_{l s_{2}}\left(g_{s_{1} l}^{C U}\right)=\frac{\alpha_{l}}{\left(2+3 \tau^{2}\right)} .
$$

Clearly, both external tariffs increase with $\alpha_{l}$. Note that, the external tariffs of CU members are independent of $\alpha_{l}$ when transportation costs are zero $(\tau=1)$. This is intuitive since a CU acts as a common market so the import demand of member countries and the export supply of the non-member to the member countries cannot be differentiated due to non-existence of transportation costs. However, as $\tau$ goes down, the export supply of the non-member country (one of the close countries) to the far member country is steeper relative to that of the close member country since the non-member faces transportation costs only when exporting to the far country. Therefore:

$$
t_{l s_{2}}\left(g_{s_{1} l}^{C U}\right)>t_{s_{1} s_{2}}\left(g_{s_{1} l}^{C U}\right) \text { when } \tau<1
$$

Finally, under a single CU between $s_{1}$ and $s_{2}$ we have:

$$
t_{s_{1} s_{2}}\left(g_{s_{1} s_{2}}^{C U}\right)=\frac{2\left(1-\alpha_{l}\right)+\tau}{\tau\left(4+\tau^{2}\right)}
$$

A similar discussion applies as under the FTA $g_{s_{1} s_{2}}$. Moreover, given $\alpha_{l} \leq \bar{\alpha}_{l}^{t}(\tau)$, it is straightforward to show tariff complementarity is present for CU members.

\footnotetext{
${ }^{16}$ The assumption that the CU maximizes the sum of national utilities is commonly employed in the literature. Issues of the delegation of tariff-setting authority and the choice of weights in the social welfare function are discussed by Gatsios and Karp (1991) and Melatos and Woodland (2007).

${ }^{17}$ Our simple formulation of a CU's tariff choice problem is intuitively appealing and in line with much of existing literature. This is true, even when countries are asymmetric and transfers are excluded (e.g. Saggi et al. (2013)). Indeed, what our results rely upon is merely that the one period CU payoff can exceed the one period FTA payorff. Nevertheless, Syropoulos (2003) has shown that the nature of the sharing rule of a $\mathrm{CU}$ with respect to tariff revenue can affect tariff preferences as well as the trade patterns of CU members in ways that can prevent the implementation of jointly optimal tariffs. An important insight of his analysis is that CU members have an incentive to influence their common tariffs not just for external terms-of-trade reasons but also for internal distributional purposes. Given the focus of our paper, we abstract from such considerations.

${ }^{18}$ In Bagwell and Staiger (1997), CU members compete for imports rather than compete for exports as in our model. In that context, a CU is only beneficial because of a "market power" effect: CU members pool their market power and extract a larger terms of trade gain from non-members.
} 


\begin{tabular}{c|c}
\hline \hline$\varnothing$ & $\varnothing, g_{i j}, g_{i k}, g_{j k}, g_{i j}^{C U}, g_{i k}^{C U}, g_{j k}^{C U}, g^{F T}$ \\
$g_{i j}^{C U}$ & $g_{i j}^{C U}, g^{F T}$ \\
$g_{i j}$ & $g_{i j}, g_{i}^{H}, g_{j}^{H}, g^{F T}$ \\
$g_{i}^{H}$ & $g_{i}^{H}, g^{F T}$ \\
$g^{F T}$ & $g^{F T}$ \\
\hline
\end{tabular}

Table 1: Networks and possible transitions within a period

Unsurprisingly, CU external tariffs always exceed those under an FTA because CU members internalize the effect of their external tariffs on each other's export surplus:

$$
t_{s_{1} l}\left(g_{s_{1} s_{2}}^{C U}\right)>t_{s_{1} l}\left(g_{s_{1} s_{2}}\right), t_{s_{1} s_{2}}\left(g_{s_{1} l}^{C U}\right)>t_{s_{1} s_{2}}\left(g_{s_{1} l}\right) \text { and } t_{l_{1}}\left(g_{s_{1} l}^{C U}\right)>t_{l_{1}}\left(g_{s_{1} l}\right)
$$

\section{Dynamic game}

\section{$3.1 \quad$ Network dynamics}

Our dynamic model is very similar to Seidmann (2009). Indeed, the set of trade agreement networks and the possible transitions between such networks are identical. Thus, we assume that at most one agreement can be formed in any given period and agreements formed in previous periods are binding. ${ }^{19,20}$ We let $g$ denote an arbitrary network with Figure 1 illustrating the possible networks. The Free trade network $g^{F T}$ could be obtained by i) a three country MFN agreement taking the world directly to global free trade from the empty network, an FTA insider-outsider or an CU insider-outsider network, or ii) a sequence of three FTAs. Given a network at the end of the previous period $g_{t-1}$, we follow Seidmann (2009) and refer to the current period $t$ as the subgame at network $g_{t-1}$. Table 1 describes the feasible transitions that can arise in a given period or, equivalently, subgame. We let $g_{t-1} \rightarrow g_{t}$ denote the (feasible) transition from $g_{t-1}$ to $g_{t}$.

Seidmann (2009) assumes a stochastic protocol regarding which country, called the "proposer", can propose an agreement in a given period. However, we assume a deterministic protocol. The basic idea of our protocol is twofold. First, the large country is the "leader

\footnotetext{
${ }^{19}$ Many authors (e.g. Ornelas (2008) and Ornelas and Liu (2012)) have argued the binding nature of trade agreements is entirely realistic and pervasive in the trade agreements literature. They argue realism in terms of real world observation and in terms of being a reduced form shorthand for a more structural modeling approach. See McLaren (2002) for sunk costs as a structural justification and Roberts and Tybout (1997), Eichengreen and Irwin (1998) and Freund and McLaren (1999) for empirical support thereof.

${ }^{20}$ Essentially, we interpret a period as the length of time taken to negotiate an agreement. Trade agreement negotiations typically take many years to complete; for example, NAFTA negotiations date back to 1986 despite not being signed in 1992 and not being implemented in 1994.
} 

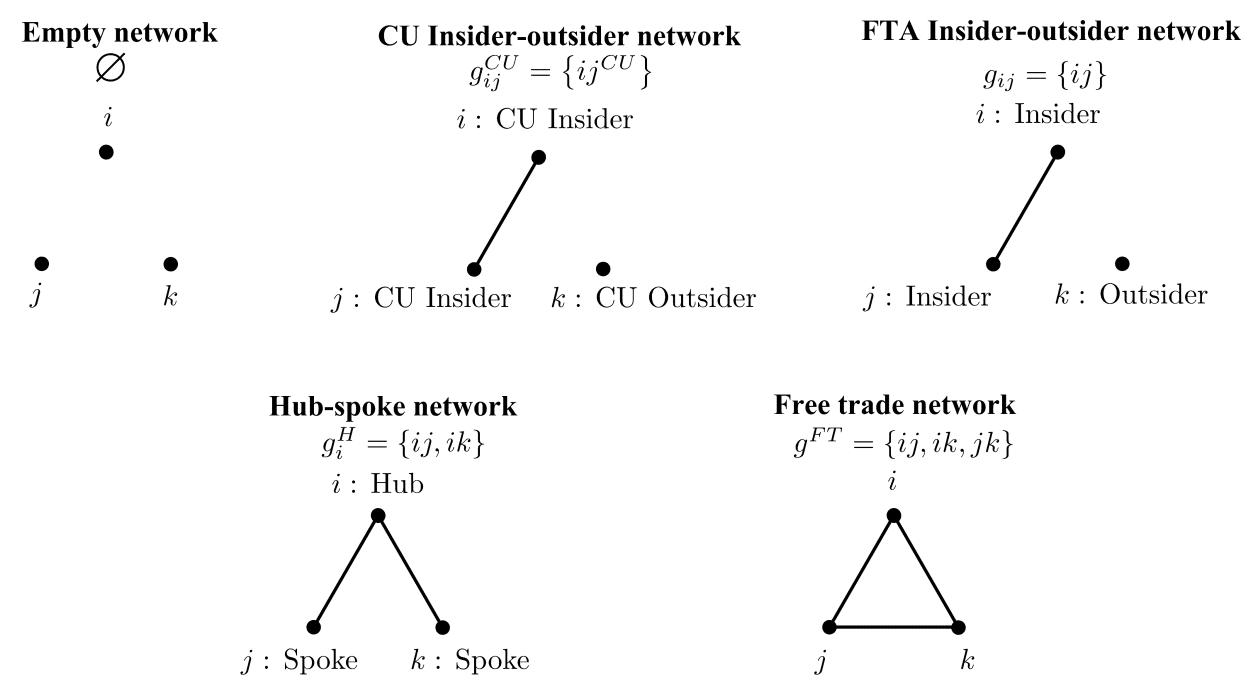

Figure 1: Networks and network positions

country" who has the first opportunity in a given period to propose agreements. However, if the small countries reject proposals made by the large country then they have an opportunity to propose agreements. After outlining our protocol, we discuss a number of reasons guiding our choice of this particular protocol. In Section 5, we demonstrate that our main results are robust to modifications of the protocol.

Our protocol consists of stages 1(a)-1(c) below. In period $t$, regardless of the stage of the protocol, a proposer can only propose an agreement in which they are a member (e.g. the proposer cannot propose that the other two countries form an FTA) and which represents a feasible transition as described in Table 1. To be clear, a proposer can choose to propose no agreement. In general, the exogenous protocol proceeds according to the following stages in each period:

- Stage 1(a): $l$ has the opportunity to propose an agreement. If all members of the proposed agreement accept the proposal, the agreement forms and the period ends. If $l$ proposes a bilateral PTA and it is rejected, the game moves to stage 1(b). If $l$ proposes $g^{F T}$ and only one small country rejects the proposal, the game moves to stage 1(b). If $l$ proposes $g^{F T}$ and both $s_{1}$ and $s_{2}$ reject, or if $l$ proposes no agreement, the game moves to stage $1(\mathrm{c})$.

- Stage 1(b): $l$ has the opportunity to propose a bilateral PTA to the small country who did not reject the proposal in stage 1(a). If an agreement forms, the period ends. If no agreement forms, the game moves to stage $1(\mathrm{c})$.

- Stage 1(c): $s_{1}$ has the opportunity to propose a bilateral PTA to $s_{2}$. No matter what happens here, the period ends. 
In general, these stages govern the protocol regarding the proposer. However, given our assumption that agreements are irreversible, some stages are redundant depending on the network at the beginning of the period. For example, stage 1(c) is redundant in period $t$ if $s_{1}$ and $s_{2}$ have a PTA at the end of period $t-1$ (e.g. $\left.g_{t-1}=g_{s_{1} s_{2}}\right)$. Additionally, for example, stages $1(\mathrm{a})$ and $1(\mathrm{~b})$ are redundant in period $t$ if $l$ has FTAs with $s_{1}$ and $s_{2}$ at the end of period $t-1$ (i.e. $g_{t-1}=g_{l}^{H}$ ). In any case, the protocol together with the assumption that agreements are irreversible implies no further agreements form once the Free trade network is obtained or no pair of countries want to form a subsequent agreement which happens in at most three periods.

In Section 5, we discuss how our main results are robust to various alternative protocols including a small country being the "leader country" or a small country being able to propose agreements involving the large country in stage 1(c). Nevertheless, a number of reasons guide our choice of the above protocol. First, the protocol is very similar in spirit to that used by Aghion et al. (2007) in their extensive form game. However, unlike Aghion et al. (2007), we allow i) the possibility of small countries being the proposer and hence forming their own PTA, and ii) the possibility of PTA formation after a small country rejects the large country's proposal of global free trade.

Second, Baier et al. (2014) find that, empirically, the order in which pairs of countries form agreements over time tends to be determined by the magnitude of gains associated with the agreement. Put simply, countries with larger joint gains from an agreement form agreements before countries with lower joint gains from an agreement. Whenever multiple agreements form in the equilibrium of our model, agreements involving the large far country yield higher joint member gains than agreements involving both small close countries. This suggests modeling the large country as the leader country.

Our third reason relates to the well known fact (Ludema (1991), Ray and Vohra (1997) and Jackson (2008)) that equilibria can be quite sensitive to exogenous protocols (whether deterministic or stochastic). Indeed, in previous versions of this paper we endogenized what agreement emerges in a given period by allowing each country to freely announce the agreement it wants to form in each period. Having defined a simultaneous move equilibrium concept to solve this "announcement" game in each period, we used backward induction to determine the "subgame perfect" path of agreements. However, to avoid existence issues, we were forced to use rather complex equilibrium concepts to solve the simultaneous move game within a period. This complexity was magnified once we embedded the simultaneous move game in a dynamic game. However, the equilibrium outcomes we now obtain under our exogenous protocol are nearly identical to those of earlier versions where who formed what agreement in a given period was completely endogenous. 
Our final reason relates to issues regarding the use of what would perhaps be the most natural alternative protocol. Arguably, such a protocol would allow a single proposer in each period with each country having equal probability of being the proposer (Seidmann (2009) does something that is closer to this stochastic protocol than our deterministic protocol). However, in general, this setup introduces complications associated with the "possibility of waiting". To illustrate, suppose $s_{1}$ is chosen as the proposer and that $s_{1}$ 's ideal outcome is a CU with $l$ but l's ideal outcome is an FTA with $s_{1}$. Then $l$ could face an intertemporal trade off when $s_{1}$ proposes a $\mathrm{CU}$ : forming a $\mathrm{CU}$ in the current period could be better than the status quo of no agreement but waiting leads to the possibility of being the proposer in the following period and forming an FTA. Of course, the "possibility of waiting" really only creates complications for intermediate values of the discount factor $\beta$ : when $\beta=0$ only myopic considerations matter and when $\beta \approx 1$ then (essentially) only the final outcome matters. Indeed, Seidmann (2009) focuses on these two cases and so waiting is not a complication. But, our central results emerge for intermediate values of $\beta$ and so we don't want this issue of waiting to drive or complicate the interpretation of our results.

\subsection{Payoffs}

As evident from Section 2, we let $W_{i}\left(g_{t}\right)$ denote country $i$ 's one period payoff given the network $g_{t}$ in period $t$. In addition to letting $\beta$ denote the discount factor, we need some more notation to describe continuation payoffs. Table 1 describes the set of networks in the current period, denoted $G\left(g_{t-1}\right)$, that are the result of feasible transitions from the network at the beginning of the current period $g_{t-1}$. Additionally, $\mathcal{G}_{t_{0}}=\left(g_{t_{0}}, g_{t_{0}+1}, g_{t_{0}+2}, \ldots\right)$ denotes a path of networks occurring from (the end of) period $t_{0}$ onwards where each network on the path results from a feasible transition in the given period. Thus, country $i$ 's continuation payoff from period $t_{0}$ onwards is $V_{i}\left(\mathcal{G}_{t_{0}}\right)=\sum_{t=t_{0}}^{\infty} \beta^{t-t_{0}} W_{i}\left(g_{t}\right)$.

Having used backward induction to solve for subgames after the subgame at $g_{t-1}$, we refer to a network $g_{t}$ as most preferred for country $i$ in the subgame at $g_{t-1}$ if it yields a higher continuation payoff for $i$ than any other feasible outcome $g_{t}^{\prime} \in G\left(g_{t-1}\right)$. Additionally, we let $g_{t} \succ_{i} g_{t}^{\prime}$ denote that country $i$ prefers $g_{t}$ over $g_{t}^{\prime}$ in the subgame at $g_{t-1}$ since $g_{t}$ yields a higher continuation payoff for $i$ than $g_{t}^{\prime}$ in the subgame at $g_{t-1}$. It is important to note that the preference relation $\succ$ is defined over paths of networks; we merely use $W_{i}\left(g_{t}\right)>W_{i}\left(g_{t}^{\prime}\right)$ to indicate country $i$ gains a higher one period payoff from $g_{t}$ compared to $g_{t}^{\prime}$.

Finally, although a slight abuse of notation, we let the last network in a network path

indicate the network that remains in place forever. For example, $\left(g_{s l}, g_{l}^{H}, g^{F T}\right)$ indicates the network path beginning at $g_{s l}$ that reaches $g^{F T}$ via the hub-spoke network $g_{l}^{H}$ and remains 
at $g^{F T}$ forever. Alternatively, the network path $g_{s l}$ indicates the network path begins at $g_{s l}$ and remains at $g_{s l}$ forever.

\subsection{Equilibrium concept}

We follow Seidmann (2009) and solve for a type of pure strategy Markov perfect equilibrium. Specifically, we use backward induction to solve for a pure strategy subgame perfect equilibrium where the proposal by the proposer and the response(s) by the respondent(s) in period $t$ only depend on history via the network in place at the end of the previous period $g_{t-1}$. Like Seidmann (2009), we focus on the equilibrium outcome (i.e. the equilibrium path of networks) in subsequent sections rather than the equilibrium strategy profile itself.

\section{Equilibrium under moderate market size asymmetry}

Many papers in the trade agreements literature begin by presenting the equilibrium when all countries are symmetric. With a sufficiently low degree of market size and geographic asymmetry, our model makes predictions consistent with the existing literature: global free trade emerges in equilibrium. However, since our main focus is on explaining the geographic characteristics of, and the prevalence of, FTAs relative to CUs, we immediately jump to considering a moderate degree of market size asymmetry.

Our definition of a moderate degree of market size asymmetry consists of a lower bound and an upper bound on $\alpha_{l}$. The upper bound, denoted $\bar{\alpha}_{l}$, is defined as the threshold value of $\alpha_{l}$ such that neither FTA nor CU formation is attractive enough to induce l's participation in liberalization once $\alpha_{l}>\bar{\alpha}_{l}$ despite the threat of being a CU outsider. ${ }^{21}$ Specifically, CU formation is not attractive because

$$
W_{l}\left(g_{s_{1} s_{2}}^{C U}\right)>\max \left\{W_{l}\left(g_{s l}^{C U}\right), W_{l}\left(g^{F T}\right)\right\} \text { for any } \tau \text { when } \alpha_{l}>\bar{\alpha}_{l}
$$

That is, $l$ prefers remaining a CU outsider over engaging in CU formation. Additionally, FTA formation is not attractive because

$$
\frac{1}{1-\beta} W_{l}\left(g_{s_{1} s_{2}}^{C U}\right)>W_{l}\left(g_{s l}\right)+\beta W_{l}\left(g_{l}^{H}\right)+\frac{\beta^{2}}{1-\beta} W_{l}\left(g^{F T}\right) \text { for any } \beta, \tau \text { when } \alpha_{l}>\bar{\alpha}_{l} \text {. }
$$

That is, despite the threat of being a CU outsider, the continuation payoff associated with

\footnotetext{
${ }^{21}$ When $\alpha_{l}>\bar{\alpha}_{l}$ it is trivial to show a permanent CU between the small countries emerges as the equilibrium regardless of $\tau$ and $\beta$. The intuition is simple: since the large country prefers remaining a permanent CU outsider over PTA formation with either small country (despite the fact it will become the hub if it engages in FTA formation), the small countries exploit the CU coordination benefit and form a CU.
} 
exploiting the FTA flexibility benefit and being the hub on the path to global free trade is not attractive enough to induce l's participation in liberalization. ${ }^{22}$

The lower bound on market size asymmetry, denoted $\underline{\alpha}_{l}$, ensures $s$ and $l$ hold a "CU exclusion incentive" whenever PTA formation is more attractive for $l$ than being a permanent CU outsider. By $s$ and $l$ holding a "CU exclusion incentive" we mean $W_{i}\left(g_{s l}^{C U}\right)>W_{i}\left(g^{F T}\right)$ for $i=s, l$. Since $s$ 's CU exclusion incentive is the binding incentive, then $\underline{\alpha}_{l}$ is the threshold value of $\alpha_{l}$ such that $W_{s}\left(g_{s l}^{C U}\right)>W_{s}\left(g^{F T}\right)$ for any $\tau$ when i) $\alpha_{l}>\underline{\alpha}_{l}$ and ii) either (22) or (23) fail. This lower bound on $\alpha_{l}$ helps streamline our analysis because it will imply $s$ 's preferred PTA with $l$ is a CU when $l$ would be the hub on a path of FTAs leading to global free trade.

To focus on the range of the parameter space where there is a moderate degree of market size asymmetry, we assume hereafter that $\underline{\alpha}_{l} \leq \alpha_{l} \leq \bar{\alpha}_{l}$. As such, our analysis focuses on the area of the parameter space where i) PTA formation is attractive enough to induce $l$ 's participation in liberalization when faced with the threat of being a permanent CU outsider and ii) $s$ and $l$ hold a CU exclusion incentive. This range of the parameter space is not trivial; in our model, it represents approximately half of the parameter space. ${ }^{23}$ We now proceed to characterize the equilibrium path of networks by using backward induction.

\subsection{Subgames at hub-spoke and insider-outsider networks}

The first step in using backward induction to solve for the equilibrium path of networks is to solve the equilibrium agreement in subgames at hub-spoke networks. This task is simple. A pair of spokes $j$ and $k$ form the final FTA leading to global free trade if and only if $W_{j}\left(g^{F T}\right)>W_{j}\left(g_{i}^{H}\right)$ and $W_{k}\left(g^{F T}\right)>W_{k}\left(g_{i}^{H}\right)$. This always holds for country $s$ but not necessarily for country $l$. $W_{l}\left(g^{F T}\right)<W_{l}\left(g_{s}^{H}\right)$ is possible because the benefit of market access to an additional small country may not compensate the large country for the preferential market access it gives up by entering an FTA. Lemma 1 summarizes these findings.

Lemma 1 Consider a subgame at the hub-spoke network $g_{i}^{H}$. If $l$ is a spoke and $W_{l}\left(g_{s}^{H}\right)>$ $W_{l}\left(g^{F T}\right)$, no agreement arises meaning s remains the hub (i.e. $\left.g_{i}^{H} \rightarrow g_{i}^{H}\right)$. Otherwise, global free trade emerges (i.e. $g_{i}^{H} \rightarrow g^{F T}$ ).

Rolling back to subgames at insider-outsider networks, the key issue is which country (if any) becomes the hub. Obviously, FTA formation here requires the consent of an insider and the outsider.

\footnotetext{
${ }^{22}$ Note, it is possible that $\bar{\alpha}_{l}$ exceeds $\bar{\alpha}_{l}^{t}(\tau)$ (see $\left.\sqrt{16}\right)$ ). In this case, our effective upper bound on $\alpha_{l}$ is $\min \left\{\bar{\alpha}_{l}, \bar{\alpha}_{l}^{t}(\tau)\right\}$

${ }^{23}$ In our model, we have the following critical values: $\underline{\alpha}_{l} \approx 1.09, \bar{\alpha}_{l} \approx 1.18$ and $\bar{\alpha}_{l}^{t}(1) \approx 1.2$. Moreover, the requirement that $\bar{\alpha}_{l}^{t}(\tau) \geq \underline{\alpha}_{l}$ yields a lower bound on $\tau$ of $\underline{\tau} \approx \frac{2}{5}$.
} 
However, an outsider may prefer to remain an outsider because of FTA free riding incentives. To avoid triviality, suppose $W_{i}\left(g^{F T}\right)>W_{i}\left(g_{k}^{H}\right)$ for each spoke country $i$ so that Lemma 1 implies $g_{k}^{H} \rightarrow g^{F T}$ for any hub country $k$. Then, $i$ prefers to become a spoke rather than remain an outsider if and only if

$$
W_{i}\left(g_{k}^{H}\right)+\frac{\beta}{1-\beta} W_{i}\left(g^{F T}\right)>\frac{1}{1-\beta} W_{i}\left(g_{j k}\right)
$$

Clearly (24) fails if $W_{i}\left(g_{j k}\right)>\max \left\{W_{i}\left(g_{k}^{H}\right), W_{i}\left(g^{F T}\right)\right\}$ which is actually true for $l$. Thus, $l$ prefers to free ride on the external tariff liberalization of the FTA between $s_{1}$ and $s_{2}$ rather than engage in subsequent FTA formation. As a result, $s_{1}$ and $s_{2}$ remain insiders forever if they become insiders. Conversely, 24 clearly holds if $W_{i}\left(g^{F T}\right)>W_{i}\left(g_{k}^{H}\right)>W_{i}\left(g_{j k}\right)$ which is actually true for $s$ as an outsider. That is, regardless of the hub's identity, a small country wants to participate in FTA formation when it is an outsider.

When $s$ and $l$ are insiders, two trade-offs drive their incentives regarding whether to become the hub. The first trade-off emerges when $l$ refuses FTA formation as a spoke. In this case, $s$ knows that it remains the hub forever upon becoming the hub. This creates the following trade off. On one hand, $l$ prefers remaining a spoke over global free trade. On the other hand, becoming the hub is attractive, even though global free trade will then follow, because $l$ gains temporary sole preferential access to the spoke markets rather than being permanently discriminated against as a spoke. Moreover, given the value placed on market access to $l$ by the outsider, the outsider prefers to form an FTA with $l$ rather than the other small country. Thus, in the case where $l$ would refuse FTA formation as a spoke, $l$ becomes the hub if and only if $W_{l}\left(g_{l}^{H}\right)+\frac{\beta}{1-\beta} W_{l}\left(g^{F T}\right)>\frac{1}{1-\beta} W_{l}\left(g_{s}^{H}\right)$ which reduces to the Free Trade-Spoke (FT-K) condition:

$$
\beta<\frac{W_{l}\left(g_{l}^{H}\right)-W_{l}\left(g_{s}^{H}\right)}{W_{l}\left(g_{l}^{H}\right)-W_{l}\left(g^{F T}\right)} \equiv \bar{\beta}_{l}^{F T-K}\left(\alpha_{l}, \tau\right) .
$$

When each country wants to engage in FTA formation as a spoke, a second trade off emerges. This second trade off arises from an "FTA exclusion incentive". By FTA exclusion incentive, we mean $W_{i}\left(g_{i j}\right)>W_{i}\left(g^{F T}\right)$ : an insider wants to permanently exclude an outsider from a trilateral FTA (which is equivalent to global free trade). On one hand, becoming the hub is attractive because it affords sole preferential access to each spoke market. On the other hand, global free trade subsequently emerges when the spokes form their own FTA which erodes the value of preferential access enjoyed as the hub. Thus, an insider $i$ prefers becoming the hub rather than remaining an insider forever if and only if $W_{i}\left(g_{i}^{H}\right)+\frac{\beta}{1-\beta} W_{i}\left(g^{F T}\right)>$ $\frac{1}{1-\beta} W_{i}\left(g_{i j}\right)$. Nevertheless, this always holds for $s$ as an insider. In turn, even if this does 
not hold for $l$ as an insider, $l$ becomes the hub anyway given the anticipation that $s$ becomes the hub if $l$ does not become the hub. That is, faced with the threat of being discriminated against as a spoke, $l$ will propose an FTA to a small country even though, ideally, $l$ wants to remain an insider. Lemma 1 summarizes these findings.

Lemma 2 Consider a subgame at an insider-outsider network $g_{i j}$. If $s_{1}$ and $s_{2}$ are insiders, no agreement emerges meaning the insider-outsider network $g_{s_{1} s_{2}}$ remains (i.e. $g_{s_{1} s_{2}} \rightarrow g_{s_{1} s_{2}}$ ). If $l$ is an insider with $s$, then the hub-spoke network $g_{l}^{H}$ emerges (i.e. $g_{s l} \rightarrow g_{l}^{H}$ ) if $\beta<$ $\bar{\beta}_{l}^{F T-K}\left(\alpha_{l}, \tau\right)$ but the hub-spoke network $g_{s}^{H}$ emerges (i.e. $\left.g_{s l} \rightarrow g_{s}^{H}\right)$ if $\beta>\bar{\beta}_{l}^{F T-K}\left(\alpha_{l}, \tau\right)$.

Before rolling back to the subgame at the empty network, we consider subgames at CU insider-outsider networks. These subgames are simple. The only possibility of further liberalization is expansion to global free trade. However, expansion requires consent of $\mathrm{CU}$ insiders and the CU outsider. Given the attractiveness of market access in the large country, $l$ and (say) $s_{1}$ have a CU exclusion incentive: $l$ and $s_{1}$ want to exclude $s_{2}$ from CU expansion given $W_{i}\left(g_{s_{1} l}^{C U}\right)>W_{i}\left(g^{F T}\right)$ for $i=s, l$. As such, there is no further liberalization if $l$ becomes a $\mathrm{CU}$ insider. While, the attractiveness of market access in country $l$ means $s_{1}$ and $s_{2}$ have no $\mathrm{CU}$ exclusion incentive when they form a CU, it also means the cost of giving domestic preferential access to $s_{1}$ and $s_{2}$ may be so great that $l$ refuses to participate in expansion of $g_{s_{1} s_{2}}^{C U}$ to $g^{F T}$. Thus, the only bilateral CU that expands to $g^{F T}$ is $g_{s_{1} s_{2}}^{C U}$ but only when $W_{l}\left(g^{F T}\right)>W_{l}\left(g_{s_{1} s_{2}}^{C U}\right)$. Lemma 3 summarizes these findings.

Lemma 3 Consider a subgame at a $C U$ insider-outsider network $g_{i j}^{C U}$. If $l$ is the $C U$ outsider and $W_{l}\left(g^{F T}\right)>W_{l}\left(g_{s_{1} s_{2}}^{C U}\right)$, global free trade emerges (i.e. $\left.g_{s_{1} s_{2}}^{C U} \rightarrow g^{F T}\right)$. Otherwise, $g_{i j}^{C U}$ remains (i.e. $g_{i j}^{C U} \rightarrow g_{i j}^{C U}$ ).

\subsection{Subgame at empty network}

In analyzing the subgame at the empty network, and thus the equilibrium path of networks, we focus our attention in the main text on the case where l's FT-K condition holds. When $l$ 's FT-K condition holds, i.e. $\beta<\min \left\{\bar{\beta}_{l}^{F T-K}\left(\alpha_{l}, \tau\right), 1\right\}, l$ becomes the hub after forming an initial FTA with $s$ because it knows $s$ will become the hub otherwise. We present the case where l's FT-K condition fails in Appendix C.

Before beginning our characterization of the equilibrium path of networks, we summarize some important preferences that countries have over outcomes in the subgame at the empty network. Given Lemmas 1,3, these are preferences over paths of networks (i.e. they are based on continuation payoffs and not one period payoffs). 
Four facets of $s$ 's preferences in the subgame at the empty network underlie our results. Three of these facets follow from the fact that $s$ can never exploit the FTA flexibility benefit and become the hub given $l$ refuses FTA expansion as an FTA outsider (Lemma 2) while $l$ becomes the hub after being an FTA insider (i.e. $\beta<\bar{\beta}^{F T-K}\left(\alpha_{l}, \tau\right)$ ). First, a (permanent) $\mathrm{CU}$ with $l$ is most preferred. Second, apart from a $\mathrm{CU}$ with $l$ or a direct move to global free trade, the only outcome $s$ may prefer over an FTA with $l$ is a CU with the other small country. Third, $s$ prefers a CU with the other small country over an FTA with the other small country or the status quo of no agreements. A final facet of $s$ 's preferences is that it prefers an FTA with the other small country over a being a permanent CU outsider. Lemma 4 summarizes these preferences.

Lemma 4 Given Lemmas 1 3, country s has the following preferences in the subgame at the empty network: i) $g_{s l}^{C U}$ is most preferred, ii) $g_{s l} \succ_{s} g$ for any $g \notin\left\{g_{s l}, g^{F T}, g_{s l}^{C U}, g_{s_{1} s_{2}}^{C U}\right\}$, iii) $g_{s_{1} s_{2}}^{C U} \succ_{s} g_{s_{1} s_{2}} \succ_{s} \varnothing$, and iv) $g_{s_{1} s_{2}} \succ_{s} g_{s l}^{C U}$.

Additionally, three facets of $l$ 's preferences in the subgame at the empty network underlie our results. First, the FTA between the small countries is most preferred: $l$ ideally wants to free ride on the FTA between $s_{1}$ and $s_{2}$. Second, $l$ holds a CU exclusion incentive. Third, $l$ prefers to participate in PTA formation rather than be a permanent CU outsider at least for some values of $\alpha_{l}$ and $\tau$. Lemma 5 summarizes these preferences.

Lemma 5 Given Lemmas 143, country $l$ has the following preferences: i) $g_{s_{1} s_{2}}$ is most preferred, ii) $W_{l}\left(g_{s l}^{C U}\right)>W_{l}\left(g^{F T}\right)$, and iii) $g \succ_{l} g_{s_{1} s_{2}}^{C U}$ for some $g \in\left\{g_{s l}, g_{s l}^{C U}\right\}$ and some values of $\alpha_{l}$ and $\tau$.

Given its preferences, $l$ faces an interesting dilemma. On one hand, free riding on an FTA between the small countries is most preferred. Moreover, $l$ may even prefer the (permanent) status quo of no agreements over its preferred PTA with $s$. On the other hand, neither of these outcomes will arise if $l$ refuses to participate in liberalization since the small countries will then find it optimal to form a CU. Thus, $l$ anticipates it will becomes a CU outsider, and not an FTA outsider, if it refuses to participate in PTA formation. Indeed, this threat of being a CU outsider can induce l's participation in PTA formation. But, what is l's preferred PTA with $s$ ?

The trade off between the FTA flexibility benefit and the CU coordination benefit determines l's preferred type of PTA with $s .{ }^{24}$ l's preferred type of PTA is an FTA if and only if

\footnotetext{
${ }^{24}$ This trade off also plays an important role in Lake (2014), although geographic asymmetry is absent there.
} 
$W_{l}\left(g_{s l}\right)+\beta W_{l}\left(g_{l}^{H}\right)+\frac{\beta^{2}}{1-\beta} W_{l}\left(g^{F T}\right)>\frac{1}{1-\beta} W_{l}\left(g_{s l}^{C U}\right)$ which, upon rearranging, becomes:

$$
\beta \underbrace{\left[\left(W_{l}\left(g_{l}^{H}\right)-W_{l}\left(g_{s l}^{C U}\right)\right)+\frac{\beta}{1-\beta}\left(W_{l}\left(g^{F T}\right)-W_{l}\left(g_{s l}^{C U}\right)\right)\right]}_{\text {FTA flexibility benefit }}-\underbrace{\left(W_{l}\left(g_{s l}^{C U}\right)-W_{l}\left(g_{s l}\right)\right)}_{\text {CU coordination benefit }}>0 .
$$

That is, $l$ prefers an FTA over a CU if and only if the FTA flexibility benefit dominates the CU coordination benefit. Unlike FTA formation, CU formation affords members the opportunity to coordinate external tariffs. Thus, $W_{l}\left(g_{s l}^{C U}\right)-W_{l}\left(g_{s l}\right)$ represents the CU coordination benefit. Unlike CU formation, FTA formation affords an insider the flexibility to become the hub and thus have sole preferential access in each spoke market. Given the spokes will then form their own FTA, the square bracketed term represents the FTA flexibility benefit.

Solving (26), the FTA flexibility benefit dominates the CU coordination benefit if and only if $\beta \in\left(\underline{\beta}^{\text {Flex }}\left(\alpha_{l}, \tau\right), \bar{\beta}^{\text {Flex }}\left(\alpha_{l}, \tau\right)\right)$. Clearly, $\underline{\beta}^{\text {Flex }}\left(\alpha_{l}, \tau\right)>0$ only if the CU coordination benefit is positive. However, this is not always true. While CU formation enables external tariff coordination, market size asymmetry between CU members drives a wedge between their ideal external tariffs. Thus, $l$ may prefer to set its external tariff independently. In this case, the FTA flexibility benefit dominates the CU coordination benefit for $\beta \in\left[0, \bar{\beta}^{\text {Flex }}\left(\alpha_{l}, \tau\right)\right)$. Conversely, the CU exclusion incentive implies $\bar{\beta}^{\text {Flex }}\left(\alpha_{l}, \tau\right)<1$. As $\beta$ approaches 1 , the part of the FTA flexibility due to having sole preferential access to each spoke market vanishes as the hub leaving $W_{l}\left(g^{F T}\right)-W_{l}\left(g_{s l}^{C U}\right)<0$. Thus, the FTA flexibility benefit dominates the $\mathrm{CU}$ coordination benefit when $\beta$ lies in an intermediate range since this places sufficient weight on the part of the FTA flexibility benefit stemming from having sole preferential access to the spoke markets as the hub.

In characterizing the equilibrium path of agreements, we break down the analysis according to the degree of geographic asymmetry.

\subsubsection{Large geographic asymmetry}

We begin with larger degrees of geographic asymmetry meaning $\tau$ is lower and the large country is further away from the small countries. To this end, define $\bar{\tau}_{1}\left(\alpha_{l}\right)$ such that any greater distance between the large and small countries is so great that, despite the prospect of being a CU outsider, the large country refuses PTA formation with the small countries:

$g_{s_{1} s_{2}}^{C U} \succ_{l} g$ for $g \in\left\{g_{s l}^{C U}, g_{s l}\right\}$ if and only if $\tau<\bar{\tau}_{1}\left(\alpha_{l}\right)$ (Figure 2 depicts all critical values of $\tau)$. Additionally, define the smaller threshold of geographic asymmetry $\bar{\tau}_{2}\left(\alpha_{l}\right)$ such that $l$ prefers (permanent) CU formation with $s$ over being a (permanent) CU outsider below this 
threshold: $W_{l}\left(g_{s l}^{C U}\right)>W_{l}\left(g_{s_{1} s_{2}}^{C U}\right)$ if and only if $\tau>\bar{\tau}_{2}\left(\alpha_{l}\right)$. Together, these critical values say the flexibility benefit afforded by FTAs, but not the coordination benefit afforded by CUs, can induce the large country's participation in PTA formation to ensure it is not a CU outsider when $\tau \in\left(\bar{\tau}_{1}\left(\alpha_{l}\right), \bar{\tau}_{2}\left(\alpha_{l}\right)\right)$.

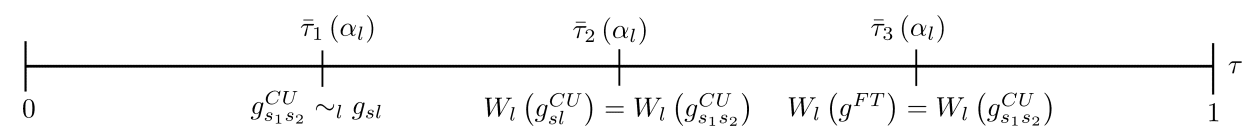

Figure 2: Critical values of transport $\operatorname{costs} \tau$

Specifically, $l$ prefers to form an FTA with $s$ rather than remain a permanent CU outsider if and only if

$$
W_{l}\left(g_{s l}\right)+\beta W_{l}\left(g_{l}^{H}\right)+\frac{\beta^{2}}{1-\beta} W_{l}\left(g^{F T}\right)>\frac{1}{1-\beta} W_{l}\left(g_{s_{1} s_{2}}^{C U}\right)
$$

Thus, analogous to 26$)$ and $\beta \in\left(\underline{\beta}^{\text {Flex }}\left(\alpha_{l}, \tau\right), \bar{\beta}^{\text {Flex }}\left(\alpha_{l}, \tau\right)\right)$, we see that 27) holds and $l$ prefers to form an FTA with $s$ if and only if $\beta \in\left(\underline{\beta}^{* \text { Flex }}\left(\alpha_{l}, \tau\right), \bar{\beta}^{* \text { Flex }}\left(\alpha_{l}, \tau\right)\right)$ because an intermediate $\beta$ places sufficient weight on the hub benefits of sole preferential access to the spoke markets. ${ }^{25}$ We now characterize the equilibrium when $\tau<\bar{\tau}_{2}\left(\alpha_{l}\right)$ which is illustrated in Figure 3 and is the main result of our paper.

Proposition 1 Suppose $\tau<\bar{\tau}_{2}\left(\alpha_{l}\right)$. The equilibrium path of networks is $\left(g_{s l}, g_{l}^{H}, g^{F T}\right)$ if $\tau \in\left(\bar{\tau}_{1}\left(\alpha_{l}\right), \bar{\tau}_{2}\left(\alpha_{l}\right)\right)$ and $\beta \in\left(\underline{\beta}^{* \text { Flex }}\left(\alpha_{l}, \tau\right), \bar{\beta}^{* \text { Flex }}\left(\alpha_{l}, \tau\right)\right)$. Otherwise, the equilibrium path of networks is $g_{s_{1} s_{2}}^{C U}$.

Proposition 1 says there exists a degree of geographic asymmetry, i.e. $\bar{\tau}_{2}\left(\alpha_{l}\right)$, above which any PTA involving the large country must be an FTA and, in this case, the large country will be the hub on the path to global free trade. Moreover, in the absence of any FTAs involving the large country, the small close countries form a CU. Since this result holds for sufficient geographic asymmetry, there is a meaningful distinction between intra and inter-regional agreements. Thus, we interpret this result as matching the striking observations that i) all CUs observed in reality are intra-regional, ii) observed FTAs are both intra and interregional and iii) the empirical observation of Chen and Joshi (2010, p.244) that the large country enjoys the benefit of overlapping FTAs because the closer countries place significant value on market access to the large country.

\footnotetext{
${ }^{25}$ Note, Lemma 3 implies $g_{s_{1} s_{2}}^{C U} \rightarrow g_{s_{1} s_{2}}^{C U}$ given that Lemma 5 and $\tau<\bar{\tau}_{2}\left(\alpha_{l}\right)$ imply $W_{l}\left(g_{s_{1} s_{2}}^{C U}\right)>$ $W_{l}\left(g_{s l}^{C U}\right)>W_{l}\left(g^{F T}\right)$.
} 


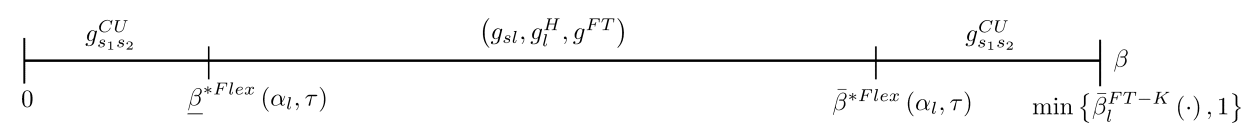

Figure 3: Equilibrium path of networks under large geographic asymmetry

The key intuition behind this equilibrium structure is that FTA formation is the only type of PTA formation attractive enough to induce l's participation in liberalization given the prospect of being permanently discriminated against as a CU outsider. The possibility that FTA formation is more attractive than CU formation emerges because of the FTA flexibility benefit: $l$ values the ability to have overlapping FTAs with the small countries (at least temporarily) because it gains sole preferential access to these markets. Conversely, $l$ cannot achieve this flexibility via a CU. Both market size and geographic asymmetry play important roles in delivering an FTA as the only PTA attractive enough to induce l's participation. As we discussed in Section 2.1.2, a rising $\alpha_{l}$ creates a bigger wedge between the tariff preferences of $l$ and $s$ and, by reducing the value of tariff coordination, makes an FTA more attractive relative to a CU. ${ }^{26}$ However, by lowering trade flows far enough, sufficient geographic asymmetry is also required to ensure $l$ prefers remaining a permanent $\mathrm{CU}$ outsider rather than engaging in $\mathrm{CU}$ formation. Thus, given our moderate degree of market size asymmetry and sufficient geographic asymmetry, the only type of PTA attractive enough to induce the large country's participation is an FTA.

Despite FTA formation representing the only way that the large will participate in liberalization, equilibrium FTA formation requires a small country prefer the inter-regional FTA with the large country over the intra-regional CU with the other small country. Given $\tau<\bar{\tau}_{2}\left(\alpha_{l}\right)$ implies $l$ refuses expansion of $g_{s_{1} s_{2}}^{C U}$ to $g^{F T}$, an inter-regional FTA not only offers $s$ preferential market access to l's market but also the eventual attainment of global free trade. Thus, $s$ prefers an inter-regional FTA that eventually leads to global free trade over an intra-regional CU.

When $l$ refuses to participate in PTA formation, the small countries form their own intra-regional PTA. This intra-regional PTA takes the form of a CU since CU formation allows the small countries to exploit the $\mathrm{CU}$ coordination benefit and $l$ will not participate in liberalization following any type of intra-regional PTA.

\footnotetext{
${ }^{26}$ Indeed, once $\alpha_{l}<\tilde{\alpha}_{l} \approx 1.08$ it is impossible that an FTA is the only type of PTA attractive enough to induce l's participation in liberalization. That is, once $\alpha_{l}<\tilde{\alpha}_{l}$, there is no value of $\tau$ such that the inequality in 22 holds but the inequality in 23 fails. Note, $\tilde{\alpha}_{l}<\underline{\alpha}_{l}$.
} 


\subsubsection{Small geographic asymmetry}

We now focus on the range of transport $\operatorname{costs} \tau>\bar{\tau}_{2}\left(\alpha_{l}\right)$. Given we are now focusing on sufficiently small degrees of geographic asymmetry, we now interpret all agreements as intra-regional.

Two issues will drive the equilibrium structure. First, the trade off between the FTA flexibility and $\mathrm{CU}$ coordination benefits will actually bind since country $l$ now prefers a permanent CU with country $s$ over being a permanent CU outsider. Second, when $l$ prefers FTA rather than CU formation with $s$, then $s$ faces a non-trivial trade off between accepting an FTA offer from $l$ or forming a CU with the other small country. This second trade off was not relevant earlier.

Before characterizing the equilibrium, we briefly discuss what makes CU formation with another small country attractive relative to FTA formation with the large country. The attractiveness stems from the expansion of $g_{s_{1} s_{2}}^{C U}$ to $g^{F T}$ which provides market access to $l$ 's market without the discrimination faced as a spoke that $s$ has to suffer after FTA formation with $l$. Indeed, given the market size of $l$, we actually have $W_{s}\left(g_{s l}\right)>W_{s}\left(g_{s_{1} s_{2}}^{C U}\right)$ meaning that, myopically, $s$ prefers FTA formation with $l$ rather than CU formation with the other small country. Thus, $s$ prefers FTA formation with $l$ over CU formation with another small country if and only if $\beta<\bar{\beta}^{s}\left(\alpha_{l}, \tau\right)$ where $\bar{\beta}^{s}\left(\alpha_{l}, \tau\right)>1$ when $g_{s_{1} s_{2}}^{C U}$ does not expand to $g^{F T} \cdot{ }^{27}$ Moreover, $l$ willingly participates in such expansion only when geographic asymmetry falls below the threshold $\bar{\tau}_{3}\left(\alpha_{l}\right): W_{l}\left(g^{F T}\right)>W_{l}\left(g_{s_{1} s_{2}}^{C U}\right)$ if and only if $\tau>\bar{\tau}_{3}\left(\alpha_{l}\right)$. Importantly, this implies $s$ cannot credibly threaten to form a CU with the other small country in the face of an FTA offer from $l$ in stages $1(\mathrm{a})$ or $1(\mathrm{~b})$ unless $\tau>\bar{\tau}_{3}\left(\alpha_{l}\right)$.

Proposition 2 now characterizes the equilibrium when the FTA flexibility benefit dominates the CU coordination benefit and is illustrated in Figure 4.

Proposition 2 Suppose $\tau>\bar{\tau}_{2}\left(\alpha_{l}\right)$ and let $\beta \in\left(\underline{\beta}^{\text {Flex }}\left(\alpha_{l}, \tau\right), \bar{\beta}^{\text {Flex }}\left(\alpha_{l}, \tau\right)\right)$ so that the FTA flexibility benefit dominates the $C U$ coordination benefit. The equilibrium path of networks is: i) $\left(g_{s l}, g_{l}^{H}, g^{F T}\right)$ unless $\tau>\bar{\tau}_{3}\left(\alpha_{l}\right)$ and $\beta>\bar{\beta}^{s}\left(\alpha_{l}, \tau\right)$, but ii) $g_{s l}^{C U}$ if $\tau>\bar{\tau}_{3}\left(\alpha_{l}\right)$ and $\beta>\bar{\beta}^{s}\left(\alpha_{l}, \tau\right)$.

Given $l$ prefers FTA over CU formation with $s$ yet $s$ prefers CU over FTA formation with $l, s$ and $l$ have different views over the type of PTA they should form. As such, $s$ would like to use the threat of forming a CU with the other small country to induce l's participation in CU rather than FTA formation. However, given our above discussion, $s$ can only credibly do so when $\tau>\bar{\tau}_{3}\left(\alpha_{l}\right)$ and, in addition, $\beta \in\left(\bar{\beta}^{s}\left(\alpha_{l}, \tau\right), \bar{\beta}_{l}^{\text {Flex }}\left(\alpha_{l}, \tau\right)\right)$. In this case, $l$ offers

\footnotetext{
${ }^{27}$ That is, $\min \left\{W_{s}\left(g_{s l}\right), W_{s}\left(g^{F T}\right)\right\}>W_{s}\left(g_{s_{1} s_{2}}^{C U}\right)$ and even though $W_{s}\left(g_{s_{1} s_{2}}^{C U}\right)>W_{s}\left(g_{l}^{H}\right)$ is possible, $g_{s l} \succ_{s} g_{s_{1} s_{2}}^{C U}$ always holds when $g_{s_{1} s_{2}}^{C U} \rightarrow g_{s_{1} s_{2}}^{C U}$.
} 
$s$ a CU which, given their CU exclusion incentive, remains forever. Otherwise, $s$ accepts an FTA offer from $l$ which then expands to global free trade via the hub-spoke network $g_{l}^{H}$.

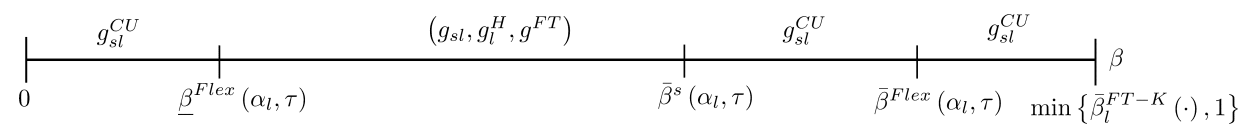

Figure 4: Equilibrium path of networks under small degree of geographic asymmetry

Proposition 2 makes clear that rising geographic asymmetry creates a discontinuity in the equilibrium structure whereby FTAs rather than CUs emerge in equilibrium. Rising geographic asymmetry reduces $l$ 's incentive to participate in expansion of $g_{s_{1} s_{2}}^{C U}$ to $g^{F T}$ and, once $\tau<\bar{\tau}_{3}\left(\alpha_{l}\right), l$ blocks this expansion. From $s$ 's perspective, this eliminates the benefit of obtaining market access to l's market without the discrimination associated with being a spoke. Thus, $\bar{\beta}^{s}\left(\alpha_{l}, \tau\right)$ jumps and we now have $\bar{\beta}^{s}\left(\alpha_{l}, \tau\right)>1$. Given $s$ can no longer credibly threaten a CU with the other small country in the face of an FTA offer from $l, s$ accepts the FTA offer from $l$ and FTA rather than CU formation occurs in equilibrium.

This role of geographic asymmetry in explaining FTA formation relative to CU formation is our second main result. Indeed, we now have two different mechanisms linking geographic asymmetry and the prevalence of FTAs relative to CUs. When geographic asymmetry is sufficiently large that agreements can be inter or intra-regional, the FTA flexibility benefit makes FTAs the only type of PTA that can induce country l's participation in liberalization (Proposition 1). This is the first mechanism. However, when geographic asymmetry is sufficiently small that all agreements are intra-regional, FTA or CU formation can be attractive enough to induce l's participation in liberalization. Moreover, when the FTA flexibility benefit dominates the $\mathrm{CU}$ coordination benefit, $l$ prefers FTA formation with $s$ while $s$ prefers $\mathrm{CU}$ formation with $l$. The key issue then becomes whether $s$ can credibly threaten a CU with the other small country as a means to engage in CU formation with $l$. The second mechanism linking geographic asymmetry and the prevalence of FTAs relative to CUs is that rising geographic asymmetry mitigates s's ability to make such credible threats. Once geographic asymmetry rises enough that $l$ refuses to participate in expansion of a CU between the small countries to global free trade, $s$ loses the ability to obtain tariff-free access to l's market without having to endure the discrimination associated with being a spoke. Thus, $s$ cannot make the credible threat needed to ensure a CU with $l$ because it actually prefers FTA formation with $l$ over CU formation with the other small country.

Proposition 3 now characterizes the equilibrium path of networks when the CU coordination benefit dominates the FTA flexibility benefit and is illustrated in Figure 4. 
Proposition 3 Suppose $\tau>\bar{\tau}_{2}\left(\alpha_{l}\right)$. Let $\beta \notin\left(\underline{\beta}^{\text {Flex }}\left(\alpha_{l}, \tau\right), \bar{\beta}^{\text {Flex }}\left(\alpha_{l}, \tau\right)\right)$ so that the $C U$ coordination benefit dominates the FTA flexibility benefit. Then, the equilibrium path of networks is $g_{s l}^{C U}$.

When the CU coordination benefit dominates the FTA flexibility benefit, a permanent $\mathrm{CU}$ between $s$ and $l$ is the equilibrium path of networks. This is not surprising. A CU with $l$ is most preferred for $s$. And, while $l$ ideally wants to free ride on an FTA between the small countries, the anticipation of a CU between the small countries induces $l$ to offer a CU to a small country. This result emphasizes the importance of the FTA flexibility benefit in explaining the prevalence of FTAs relative to CUs.

\section{Discussion}

Naturally, one might wonder how our results depend on particular modeling choices. Specifically, the choices regarding the pattern of geography and the protocol governing the order in which countries can propose agreements. We now tackle these issues in turn.

\subsection{Alternative pattern of geography}

In previous sections we assumed that the large country was the far country. But, we now assume that a small country, $s_{1}$, is the far country while the large country $l$ and the small country $s_{2}$ are the close countries. That is, $\tau_{s_{2} l}=\tau_{l s_{2}}=0$ but $\tau_{s_{1} i}=\tau_{i s_{1}}=\tau$ for $i=s_{2}, l$. We also assume that, as an outsider, the small far country $s_{1}$ prefers engaging in sequential FTA formation with $l$ and $s_{2}$ that leads to global free trade rather than remaining a permanent FTA outsider: $W_{s_{1}}\left(g^{F T}\right)>W_{s_{1}}\left(g_{l}^{H}\right)>W_{s_{1}}\left(g_{s_{2} l}\right)$.

Under this alternative pattern of geography, we obtain a starker result in terms of how geographic asymmetry affects the equilibrium type of PTA (see Appendix D for welfare expressions and proof).

Proposition 4 There is a range of moderate market size asymmetry $\left(\underline{\alpha}_{l}^{\prime}, \bar{\alpha}_{l}^{\prime}\right)$ and a critical value of geographic asymmetry $\bar{\tau}^{\prime}\left(\alpha_{l}\right)$ such that $\tau<\bar{\tau}^{\prime}\left(\alpha_{l}\right)$ implies the unique equilibrium path of networks is either a path of FTAs or an immediate move to global free trade.

In Proposition 1 we found that, under sufficient geographic asymmetry, CUs were intraregional while FTAs were both inter and intra-regional. Which of the two cases emerged in equilibrium depended on $\beta$. However, Proposition 4 says that, regardless of $\beta$, CUs never emerge when there is sufficient geographic asymmetry. 
The stronger result arises primarily because the different pattern of geography changes country l's trade off between the FTA flexibility benefit and the CU coordination benefit. Before discussing this change, it is important to note that l's trade off relates to PTA formation with the far small country $s_{1}$ because a falling $\tau$ (i.e. rising geographic asymmetry) makes PTA formation with the far country relatively more attractive than PTA formation with the close country. As $\tau$ falls, trade barriers effectively rise, and thus trade flows fall, between the close countries and the far country. Thus, giving preferential access to the far country becomes less costly. Moreover, close countries already have effective preferential access to each other, but face high effective barriers in the far country. Thus, gaining preferential market access to the far country becomes more attractive.

Unlike the case where $l$ is the far country, the FTA flexibility benefit now dominates the $\mathrm{CU}$ coordination benefit regardless of $\beta$ and this irrelevance of $\beta$ makes Proposition $4 \mathrm{a}$ starker result than Proposition 1. This follows from two observations. First, a falling $\tau$ (and a rising $\alpha_{l}$ ) creates a growing wedge between the tariff preferences of $s_{1}$ and $l$ to the extent that the CU coordination benefit is actually negative (i.e. $W_{l}\left(g_{s_{1} l}^{C U}\right)-W_{l}\left(g_{s_{1} l}\right)<0$ ). This negative CU coordination benefit is possible when $l$ is the far country but the possibility is mitigated because, as we discussed in Section 2.1.2, a falling $\tau$ initially brings the tariff preferences of $s_{1}$ and $l$ closer together. Second, unlike now, l's CU exclusion incentive as the large far country devalued the FTA flexibility benefit (see (26)) and this CU exclusion incentive strengthened as $\tau$ fell by eroding l's effective preferential access to a close country. But, here, $l$ does not hold a CU exclusion incentive: a falling $\tau$ makes $s_{1}$ more isolated and thereby increases the amount of trade, and the value of tariff-free trade, between $l$ and $s_{2}$.

Even though $l$ prefers FTA formation over CU formation with the small far country $s_{1}$ for any $\beta$ in Proposition 4, we need to make sure $s_{1}$ will accept an FTA offer from $l$ and it is optimal for $l$ to offer an FTA in order for FTA formation to arise. ${ }^{28}$ Since both small countries prefer CU rather than FTA formation with each other, the small countries will form a $\mathrm{CU}$ in stage 1(c). Thus, accepting an FTA offer from $l$ is optimal for $s_{1}$ in stage 1(a) if it prefers this FTA offer over CU formation with $s_{2}$ in stage 1(c) (or being a PTA outsider in stage 1(b)). In turn, given the threat of being a CU outsider, it is optimal for $l$ to offer an FTA to $s_{1}$ if it prefers this FTA over being a CU outsider. Both are true when $\tau<\bar{\tau}^{\prime}\left(\alpha_{l}\right)$. As $s_{1}$ becomes more isolated, and trade flows with the close countries shrink, having preferential access to the larger market of $l$ rather than the smaller market of $s_{2}$ becomes more valuable. At the same time, the reduced trade flows make the discrimination

\footnotetext{
${ }^{28} \mathrm{In}$ addition, we need to make sure that $g_{s_{1} l} \succ_{l} g^{F T}$. This is true if and only if $W_{l}\left(g_{s_{1} l}\right)+\beta W_{l}\left(g_{l}^{H}\right)+$ $\frac{\beta^{2}}{1-\beta} W_{l}\left(g^{F T}\right)>\frac{1}{1-\beta} W_{l}\left(g^{F T}\right)$ which reduces to $\beta>\frac{W_{l}\left(g^{F T}\right)-W_{l}\left(g_{s_{1} l}\right)}{W_{l}\left(g_{l}^{H}\right)-W_{l}\left(g^{F T}\right)} \equiv \tilde{\beta}_{l, s_{1}}\left(\alpha_{l}, \tau\right)$. In the proof, we show that a direct move to global free trade arises if $\beta<\tilde{\beta}_{l, s_{1}}\left(\alpha_{l}, \tau\right)$.
} 
faced as a spoke less costly. Moreover, as $\tau$ falls, the effective rise in trade barriers between the close countries and $s_{1}$ increase the value to a close country of being the country who has sole preferential access to $s_{1}$.

\section{$5.2 \quad$ Alternative protocols}

As we noted in Section 3.1, it is well known that the equilibrium of sequential move games can be very sensitive to the exogenous protocol imposed. However, our main results are quite robust to various perturbations of our protocol.

Our first main result is that all CUs are intra-regional yet FTAs are both inter and intraregional. This result arises because i) the small countries have the opportunity of forming their own CU, ii) an FTA is the only type of PTA attractive enough to induce the large country's participation in liberalization when faced with the threat of being a CU outsider, and iii) country $s$ prefers FTA formation with country $l$ over a permanent $\mathrm{CU}$ with the other small country. Thus, the features of the protocol facilitating our result are i) it affords small countries the opportunity of forming a CU and ii) a small and large country have the opportunity to form an FTA. Therefore, our main result is robust to various protocols incorporating these features.

Our second main result relates to how rising geographic asymmetry increases the prevalence of FTAs when all agreements are intra-regional. In particular, greater geographic asymmetry reduces the ability of a small country to credibly threaten a large country with being discriminated against under a small-small CU (in what follows we will just refer to this as the credible threat). The equilibrium structure where this played out was that a (permanent) small-large CU emerged when $s$ has a credible threat but a small-large FTA emerges when $s$ does not have a credible threat and this FTA is followed by a sequence of FTAs leading to global free trade. This result is robust to various tweaks of the protocol.

It is trivial to verify each alternative protocol can only, potentially, affect the subgame at the empty network and so we focus our discussion of alternative protocols on this subgame.

\section{i) $s_{1}$ can propose agreements involving $l$.}

This alternative protocol addresses concerns that $l$ may have a "last mover advantage" or "ultimatum power" in that $s_{1}$ is unable to propose agreements involving $l$ in stage 1(c). However, what really matters here is l's ability to essentially force the small countries to compete against each other in stages $1(\mathrm{a})$ and 1(b).

Remember, $s$ and $l$ have a CU exclusion incentive and, thus, the ability to propose $g^{F T}$ (which is an agreement involving $l$ ) loses relevance. When $s$ has a credible threat, then a small-large CU emerges either because $l$ offers a CU to a small country in stage 1(a) and/or 
stage $1(\mathrm{~b})$ or $s_{1}$ offers $l$ a CU in stage $1(\mathrm{c})$. When $s$ does not have a credible threat, a small-large FTA emerges because $l$ can offer an FTA to $s_{1}$ in stage 1(a) and $s_{1}$ will accept the offer given it correctly anticipates an FTA between $s_{2}$ and $l$ in stage 1(b). $s_{2}$ accepts such the FTA offer in stage 1(b) because it prefers this over being a CU insider or CU outsider which is what will happen in stage in stage $1(\mathrm{c})$.

\section{ii) $s_{1}$ rather than $l$ is the "leader" country.}

This alternative protocol addresses concerns that $l$ may have a "first mover advantage". However, what really matters here is merely whether $s_{1}$ has a better "outside option" than forming an FTA with $l$ (in our context, $s_{1}$ 's best outside option is a small-small CU).

In stage 1(c), $l$ will offer either its preferred PTA with $s$, which is an FTA, or no agreement given that $g_{s l} \succ_{s} \varnothing$. When $s_{1}$ has a credible threat it can offer $l$ a CU in stage 1(a) which $l$ will accept given $l$ correctly anticipates a small-small $\mathrm{CU}$ in stage $1(\mathrm{~b})$. A small-small CU emerges in stage $1(\mathrm{~b})$ because $g_{s_{1} s_{2}}^{C U} \succ_{s} g_{s l} \succ_{s} \varnothing$. Conversely, when $s_{1}$ does not have a credible threat then an FTA between $s$ and $l$ emerges either because $l$ rejects any proposal in stages $1(\mathrm{a})$ or $1(\mathrm{~b})$ and then offers $s$ an FTA in stage 1(c) or $s_{1}$ offers $l$ an FTA in stage $1(\mathrm{a})$.

iii) $s_{1}$ rather than $l$ is the "leader" country and $l$ can propose agreements including $s_{1}$

Given the symmetry between $s_{1}$ and $s_{2}$, the same logic applies as for the protocol just discussed in ii).

We have now demonstrated that our main results and the intuition driving our main results are robust to an alternative pattern of geography and alternative protocols.

\section{Conclusion}

We began our paper by describing two striking but often overlooked characteristics of PTA formation: i) unlike FTAs which are both inter and intra-regional, CUs are only intraregional and ii) FTAs are far more prevalent than CUs. Our model provides mechanisms that help explain these observations and these mechanisms fundamentally rely on the model's dynamic nature.

With sufficient geographic asymmetry, a meaningful distinction between intra and interregional agreements exists. We show that when there is a moderate degree of market size asymmetry, there is a degree of geographic asymmetry above which the unique equilibrium is either an intra-regional CU or a path of inter followed by intra-regional FTAs. This matches the first overlooked characteristic just described. This result rests on the fact that, in this range of the parameter space, an FTA is the only type of PTA attractive enough to 
induce the large country's participation in liberalization when faced with the threat of being a permanent $\mathrm{CU}$ outsider. In turn, this results from an inherent dynamic flexibility benefit of FTAs in that, unlike individual CU members, individual FTA members have the flexibility to form their own subsequent agreements. As such, a path of inter followed by intra-regional FTAs emerge when the large country participates in liberalization which happens when this FTA flexibility is large enough. Otherwise, an intra-regional CU emerges.

This explanation also helps explain why FTAs are so prevalent relative to CUs which is the second overlooked characteristic of PTA formation described above. However, the model provides a separate mechanism linking geographic asymmetry and FTA prevalence when geographic asymmetry is below the threshold described above. Given the low degree of geographic asymmetry here, we interpret all agreements in this area of the parameter space as intra-regional. Since the large country now prefers FTA or CU formation over being a CU outsider, the mechanism here revolves around how rising geographic asymmetry mitigates a small country's ability to credibly threaten the large country into forming a small-large CU rather than the large countries desired outcome of a small-large FTA. The small country can credibly threaten the large country with being discriminated against as a CU outsider and thus ensure a (permanent) small-large CU emerges when the small country prefers a small-small CU over a small-large FTA. However, the attractiveness of a small-small CU for a small country lies in its expansion to global free trade and obtaining access to the large country market without having to endure discrimination as a spoke. Since rising geographic asymmetry reduces the large country's incentive to participate in global free trade as a CU outsider, it mitigates a small country's ability to credibly threaten the large country into forming a small-large CU. In doing so, rising geographic asymmetry helps explain the prevalence of FTAs relative to CUs when all agreements are intra-regional.

\section{Appendix}

\section{A Welfare levels}

We report welfare levels for country $i$ under different networks $g$ as a function of an arbitrary tariff vector $\mathbf{t}^{g}$ where, for any country $i, \mathbf{t}^{g}=\left(t_{i j}^{g}, t_{i k}^{g}\right)$. With a slight abuse of notation, we let (for example) $t_{i k}^{g} \equiv t_{i k}(g)$. Note, these expressions assume the large country is the far country. See Appendix $\mathrm{D}$ for the case where the large country is a close country.

$$
W_{i}(g)=\sum_{z} C S_{i}^{z}(g)+\sum_{z} P S_{i}^{z}(g)+T R_{i}(g), i=s, l
$$


where

$$
\begin{aligned}
& \sum_{z} C S_{s_{1}}^{z}(g)=\frac{1}{2}\left[1-\tau\left(\frac{\tau^{2}\left(t_{l s_{1}}^{g}+t_{l s_{2}}^{g}\right)+\alpha_{l}}{\left(1+2 \tau^{2}\right)}-t_{l s_{1}}(g)\right)\right]^{2}+\frac{1}{2}\left[1-\frac{\tau^{2} t_{s_{1} l}^{g}+1+t_{s_{1} s_{2}}^{g}+\tau\left(\alpha_{l}-1\right)}{\left(2+\tau^{2}\right)}\right]^{2} \\
& +\frac{1}{2}\left[1-\frac{\tau^{2} t_{s_{2} l}(g)+1+t_{s_{1} s_{2}}^{g}+\tau\left(\alpha_{l}-1\right)}{\left(2+\tau^{2}\right)}+t_{s_{2} s_{1}}^{g}\right]^{2} \\
& \sum_{z} C S_{l}^{z}(g)=\frac{1}{2}\left[\alpha_{l}-\frac{\tau^{2}\left(t_{l s_{1}}^{g}+t_{l s_{2}}^{g}\right)+\alpha_{l}}{\left(1+2 \tau^{2}\right)}\right]^{2}+\frac{1}{2}\left[\alpha_{l}-\tau\left(\frac{\tau^{2} t_{s_{1} l}^{g}+\tau\left(\alpha_{l}-1\right)+1+t_{s_{1} s_{2}}^{g}}{\left(2+\tau^{2}\right)} t_{s_{1} l}^{g}\right)\right]^{2} \\
& +\frac{1}{2}\left[\alpha_{l}-\tau\left(\frac{\tau^{2} t_{s_{2} l}^{g}+\tau\left(\alpha_{l}-1\right)+1+t_{s_{2} s_{1}}^{g}}{\left(2+\tau^{2}\right)}-t_{s_{2} l}^{g}\right)\right]^{2} \\
& \sum_{z} P S_{s_{1}}^{z}(g)=\frac{1+\tau\left(\alpha_{l}-1\right)-t_{s_{2} s_{1}}^{g}+\tau^{2}\left(t_{s_{2} l}^{g}-t_{s_{2} s_{1}}^{g}\right)}{\left(2+\tau^{2}\right)}+\tau \frac{\alpha_{l}-t_{l s_{1}}^{g}+\tau^{2}\left(t_{l s_{2}}^{g}-t_{l s_{1}}^{g}\right)}{\left(1+2 \tau^{2}\right)} \\
& \sum_{z} P S_{l}^{z}(g)=\tau\left[\frac{1+\tau\left(\alpha_{l}-1\right)+t_{s_{1} s_{2}}^{g}-2 t_{s_{1} l}^{g}}{\left(2+\tau^{2}\right)}+\frac{1+\tau\left(\alpha_{l}-1\right)+t_{s_{2} s_{1}}^{g}-2 t_{s_{2} l}^{g}}{\left(2+\tau^{2}\right)}\right]
\end{aligned}
$$

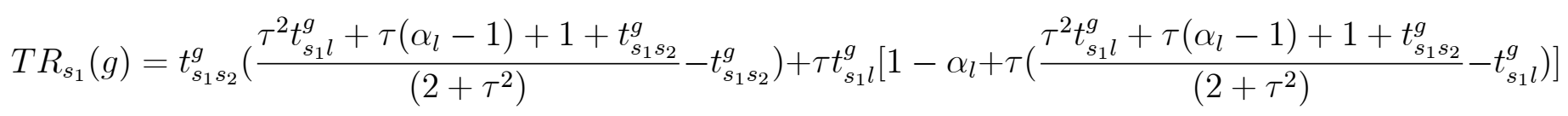

$$
\begin{aligned}
& T R_{l}(g)=\tau^{2} t_{l s_{1}}^{g}\left(\frac{\tau^{2}\left(t_{l s_{1}}^{g}+t_{l s_{2}}^{g}\right)+\alpha_{l}}{\left(1+2 \tau^{2}\right)}-t_{l s_{1}}^{g}\right)+\tau^{2} t_{l s_{2}}^{g}\left(\frac{\tau^{2}\left(t_{l s_{1}}^{g}+t_{l s_{2}}^{g}\right)+\alpha_{l}}{\left(1+2 \tau^{2}\right)}-t_{l s_{2}}^{g}\right)
\end{aligned}
$$

Using the welfare equations reported above and tariff levels reported in the text, we can easily obtain the formulae for welfare levels under all possible networks.

\section{B Proofs}

Proof of Lemma 1

Before beginning we note that $W_{s}\left(g^{F T}\right)>W_{s}\left(g_{i}^{H}\right)$ for $i=l$ or $i=s^{\prime} \neq s$ because $f_{1 i}\left(\tau, \alpha_{l}\right)=W_{s_{1}}\left(g^{F T}\right)-W_{s_{1}}\left(g_{i}^{H}\right)$ is increasing in $\alpha_{l}$ and minimized at $f_{1 i}\left(1, \underline{\alpha}_{l}\right)>0$.

If $g=g_{l}^{H}$, then stages $1(\mathrm{a})$ and $1(\mathrm{~b})$ are redundant. In stage $1(\mathrm{c}), W_{i}\left(g^{F T}\right)>W_{i}\left(g_{l}^{H}\right)$ for $i=s_{1}, s_{2}$ implies $s_{2}$ will accept a proposed FTA from $s_{1}$ and, in turn, it is optimal for $s_{1}$ to propose an FTA with $s_{2}$. Thus, $g_{l}^{H} \rightarrow g^{F T}$ arises.

If $g=g_{s_{1}}^{H}$, then stage $1(\mathrm{~b})$ and $1(\mathrm{c})$ are redundant. In stage $1(\mathrm{a}), W_{s_{2}}\left(g^{F T}\right)>W_{s_{2}}\left(g_{s_{1}}^{H}\right)$ implies $s_{2}$ will accept a proposed FTA from $l$ and, in turn, it is optimal for $l$ to propose an FTA with $s_{2}$ iff $W_{l}\left(g^{F T}\right)>W_{l}\left(g_{s_{1}}^{H}\right)$. Thus, by symmetry, $g_{s}^{H} \rightarrow g^{F T}$ arises iff $W_{s_{2}}\left(g^{F T}\right)>$ 
$W_{s_{2}}\left(g_{s_{1}}^{H}\right)$ with $g_{s}^{H} \rightarrow g_{s}^{H}$ arising otherwise.

Proof of Lemma 2

Before beginning we note that $W_{l}\left(g_{s_{1} s_{2}}\right)>\max \left\{W_{l}\left(g_{s}^{H}\right), W_{l}\left(g^{F T}\right)\right\}$ and $W_{s}\left(g^{F T}\right)>$ $W_{s}\left(g_{i}^{H}\right)>W_{s}\left(g_{s^{\prime} l}\right)$ for $i=l$ or for $i=s^{\prime} \neq s$. This follows because $f_{21}\left(\tau, \alpha_{l}\right)=$ $W_{l}\left(g_{s_{1} s_{2}}\right)-W_{l}\left(g_{s}^{H}\right), f_{22}\left(\tau, \alpha_{l}\right)=W_{l}\left(g_{s_{1} s_{2}}\right)-W_{l}\left(g^{F T}\right), f_{23}\left(\tau, \alpha_{l}\right)=W_{s_{1}}\left(g^{F T}\right)-W_{s_{1}}\left(g_{s_{2} l}\right)$, $f_{24}\left(\tau, \alpha_{l}\right)=W_{s_{1}}\left(g_{l}^{H}\right)-W_{s_{1}}\left(g_{s_{2} l}\right)$ and $f_{25}\left(\tau, \alpha_{l}\right)=W_{s_{1}}\left(g_{s_{2}}^{H}\right)-W_{s_{1}}\left(g_{s_{2} l}\right)$ are increasing in $\alpha_{l}$ and minimized at $f_{2 n}\left(1, \underline{\alpha}_{l}\right)>0$ for $n=1,2,3,4,5$.

If $g_{i j}=g_{s_{1} s_{2}}$, then stage 1 (c) is redundant. Thus, $g_{s_{1} s_{2}} \rightarrow g_{s_{1} s_{2}}$ arises if $l$ proposes no agreement in stage 1 (a). Indeed, this is optimal for $l$ given $W_{l}\left(g_{s_{1} s 2}\right)>\max \left\{W_{l}\left(g_{s}^{H}\right), W_{l}\left(g^{F T}\right)\right\}$.

Now suppose $g_{i j}=g_{s_{1} l}$. Country $i=s_{1}, l$ prefers to become the hub rather than remain an insider forever iff $W_{i}\left(g_{i}^{H}\right)+\frac{\beta}{1-\beta} W_{i}\left(g^{F T}\right)>\frac{1}{1-\beta} W_{i}\left(g_{i j}\right)$ which reduces to $\beta<\hat{\beta}_{i}\left(\alpha_{l}, \tau\right) \equiv$ $\frac{W_{i}\left(g_{i}^{H}\right)-W_{i}\left(g_{i j}\right)}{W_{i}\left(g_{i}^{H}\right)-W_{i}\left(g^{F T}\right)}$. Moreover, $\hat{\beta}_{s}\left(\alpha_{l}, \tau\right)>\max \left\{\hat{\beta}_{l}\left(\alpha_{l}, \tau\right), \bar{\beta}_{l}^{F T-K}\left(\alpha_{l}, \tau\right)\right\}$ because $f_{26}\left(\tau, \alpha_{l}\right)=$ $\hat{\beta}_{s}\left(\alpha_{l}, \tau\right)-\hat{\beta}_{l}\left(\alpha_{l}, \tau\right)$ and $f_{27}\left(\tau, \alpha_{l}\right)=\hat{\beta}_{s}\left(\alpha_{l}, \tau\right)-\bar{\beta}_{l}^{F T-K}\left(\alpha_{l}, \tau\right)$ are decreasing in $\tau$ and minimized at $f_{2 n}\left(1, \bar{\alpha}_{l}\right)>0$ for $n=6,7$. Thus, regardless of $\beta$, it is optimal for $s_{1}$ to propose an FTA with $s_{2}$ and, given $W_{s_{2}}\left(g^{F T}\right)>W_{s_{2}}\left(g_{s_{1}}^{H}\right)>W_{s_{2}}\left(g_{s_{1} l}\right)$, it is optimal for $s_{2}$ to accept the FTA in stage 1(c). By symmetry, the same is true for $g_{i j}=g_{s_{2} l}$. If stage 1(b) arises, it is optimal for a small country (say $s_{2}$ ) to accept a proposed FTA from $l$ given $W_{s_{2}}\left(g^{F T}\right)>W_{s_{2}}\left(g_{l}^{H}\right)>W_{s_{2}}\left(g_{s_{1} l}\right)$. In turn, it is optimal for $l$ to propose an FTA with $s_{2}$ in stage $1(\mathrm{~b})$ iff $\beta<\bar{\beta}_{l}^{F T-K}\left(\alpha_{l}, \tau\right)$. By similar logic, it is optimal for $l$ to propose an FTA with $s_{2}$ in stage 1 (a) iff $\beta<\bar{\beta}_{l}^{F T-K}\left(\alpha_{l}, \tau\right)$. Thus, by symmetry, $g_{s l} \rightarrow g_{l}^{H}$ arises if $\beta<\bar{\beta}_{l}^{F T-K}\left(\alpha_{l}, \tau\right)$ and $g_{s l} \rightarrow g_{s}^{H}$ arises if $\beta>\bar{\beta}_{l}^{F T-K}\left(\alpha_{l}, \tau\right)$.

\section{Proof of Lemma 3}

Since the only possible expansion from $g_{i j}^{C U}$ is to $g^{F T}$, stages 1(b) and 1(c) are redundant. If $g_{i j}^{C U}=g_{s l}^{C U}$, then $g_{s l}^{C U} \rightarrow g_{s l}^{C U}$ arises in stage 1(a) because $\alpha_{l}>\underline{\alpha}_{l}$ implies $W_{l}\left(g_{s l}^{C U}\right)>$ $W_{l}\left(g^{F T}\right)$ and, thus, $l$ proposes no agreement. If $g_{i j}^{C U}=g_{s_{1} s_{2}}^{C U}$, then $s_{1}$ and $s_{2}$ will accept a proposal of $g^{F T}$ from $l$ because $W_{s}\left(g^{F T}\right)>W_{s}\left(g_{s_{1} s_{2}}^{C U}\right)$ given $f_{31}\left(\tau, \alpha_{l}\right)=W_{s}\left(g^{F T}\right)-$ $W_{s}\left(g_{s_{1} s_{2}}^{C U}\right)$ is increasing in $\alpha_{l}$ and minimized at $f_{31}\left(1, \underline{\alpha}_{l}\right)>0$. Thus, $l$ proposes $g^{F T}$ if $W_{l}\left(g^{F T}\right)>W_{l}\left(g_{s_{1} s_{2}}^{C U}\right)$ and $g_{s_{1} s_{2}}^{C U} \rightarrow g^{F T}$ arises. Otherwise, $l$ proposes no agreement and $g_{s_{1} s_{2}}^{C U} \rightarrow g_{s_{1} s_{2}}^{C U}$ arises.

Proof of Lemma 4

Part ii): $g_{s_{1} l} \succ_{s_{1}} g_{s_{2} l}$ given $f_{41}\left(\tau, \alpha_{l}\right)=W_{s_{1}}\left(g_{s_{1} l}\right)-W_{s_{1}}\left(g_{s_{2} l}\right)$ is increasing in $\alpha_{l}$ and minimized at $f_{41}\left(1, \underline{\alpha}_{l}\right)>0 . \quad g_{s_{1} l} \succ_{s_{1}} g_{s_{1} s_{2}}$ given $f_{42}\left(\tau, \alpha_{l}\right)=W_{s_{1}}\left(g_{s_{1} l}\right)-W_{s_{1}}\left(g_{s_{1} s_{2}}\right)$, $f_{43}\left(\tau, \alpha_{l}\right)=W_{s_{1}}\left(g_{l}^{H}\right)-W_{s_{1}}\left(g_{s_{1} s_{2}}\right)$ and $f_{44}\left(\tau, \alpha_{l}\right)=W_{s_{1}}\left(g^{F T}\right)-W_{s_{1}}\left(g_{s_{1} s_{2}}\right)$ are increasing in $\alpha_{l}$ and minimized at $f_{42}\left(\underline{\tau}, \bar{\alpha}_{l}\right)>0$ and $f_{4 n}\left(1, \underline{\alpha}_{l}\right)>0$ for $n=3,4 . g_{s_{1} l} \succ_{s_{1}} g_{s_{2} l}^{C U}$ given $g_{s_{1} l} \succ_{s_{1}} g_{s_{1} s_{2}}$ and $f_{45}\left(\tau, \alpha_{l}\right)=W_{s_{1}}\left(g_{s_{1} s_{2}}\right)-W_{s_{1}}\left(g_{s_{2} l}^{C U}\right)$ is increasing in $\alpha_{l}$ and minimized 
at $f_{45}\left(\underline{\tau}, \bar{\alpha}_{l}\right)>0 . g_{s_{1} l} \succ_{s_{1}} \varnothing$ given $g_{s_{1} l} \succ_{s_{1}} g_{s_{1} s_{2}}$ and $f_{46}\left(\tau, \alpha_{l}\right)=W_{s_{1}}\left(g_{s_{1} s_{2}}\right)-W_{s_{1}}(\varnothing)$ is decreasing in $\tau$ and minimized at $f_{46}\left(1, \bar{\alpha}_{l}\right)>0$.

Part iii): $f_{46}\left(\tau, \alpha_{l}\right)$ is minimized at $f_{46}\left(1, \bar{\alpha}_{l}\right)>0$ while $f_{47}\left(\tau, \alpha_{l}\right)=W_{s_{1}}\left(g_{s_{1} s_{2}}^{C U}\right)-$ $W_{s_{1}}\left(g_{s_{1} s_{2}}\right)$ is increasing in $\alpha_{l}$ and $\tau$ and minimized at $f_{47}\left(\underline{\tau}, \underline{\alpha}_{l}\right)>0$.

Part iv): $f_{44}\left(\tau, \alpha_{l}\right)$ is minimized at $f_{44}\left(1, \underline{\alpha}_{l}\right)>0$ and $f_{45}\left(\tau, \alpha_{l}\right)$ minimized is at $f_{45}\left(\underline{\tau}, \bar{\alpha}_{l}\right)>$ 0 .

Part i): Given $\beta<\bar{\beta}_{l}^{F T-K}\left(\alpha_{l}, \tau\right), \alpha_{l}>\underline{\alpha}_{l}$ and the proof of Lemma 3, $W_{s}\left(g_{s l}^{C U}\right)>$ $W_{s}\left(g^{F T}\right)>W_{s}\left(g_{s_{1} s_{2}}^{C U}\right)$. Thus, $g_{s l}^{C U} \succ_{s} g$ for $g=g^{F T}, g_{s_{1} s_{2}}^{C U}$. Given $\alpha_{l}>\underline{\alpha}_{l}$ and the proof of Lemma 2, we have $W_{s}\left(g_{s l}^{C U}\right)>\max \left\{W_{s}\left(g^{F T}\right), W_{s}\left(g_{l}^{H}\right)\right\}$. Thus, $g_{s l}^{C U} \succ_{s} g_{s l}$ because $f_{48}\left(\tau, \alpha_{l}\right)=W_{s}\left(g_{s l}^{C U}\right)-W_{s}\left(g_{s l}\right)$ is increasing in $\alpha_{l}$ and $\tau$ and minimized at $f_{48}\left(\underline{\tau}, \underline{\alpha}_{l}\right)>0$. Hence, $g_{s l}^{C U}$ is most preferred for $s$.

Proof of LeMma 5

Part iii): Follows by definition of $\bar{\alpha}_{l}$.

Part ii): Follows by definition of $\underline{\alpha}_{l}$.

Part i): $g_{s_{1} s_{2}} \succ_{l} g^{F T}$ given the proof of Lemma 3. $g_{s_{1} s_{2}} \succ_{l} \varnothing$ given $f_{51}\left(\tau, \alpha_{l}\right)=$ $W_{l}\left(g_{s_{1} s_{2}}\right)-W_{l}(\varnothing)$ is increasing in $\tau$ and minimized at $f_{51}\left(\underline{\tau}, \bar{\alpha}_{l}\right)>0 . g_{s_{1} s_{2}} \succ_{l} g_{s_{1} s_{2}}^{C U}$ given $g_{s_{1} s_{2}} \succ_{l} g^{F T}$ and $f_{52}\left(\tau, \alpha_{l}\right)=W_{l}\left(g_{s_{1} s_{2}}\right)-W_{l}\left(g_{s_{1} s_{2}}^{C U}\right)$ is increasing in $\tau$ and minimized at $f_{52}\left(\underline{\tau}, \bar{\alpha}_{l}\right)>0 . \quad g_{s_{1} s_{2}} \succ_{l} g_{s_{1} l}^{C U}$ given $g_{s_{1} s_{2}} \succ_{l} g^{F T}$ and $f_{53}\left(\tau, \alpha_{l}\right)=W_{l}\left(g_{s_{1} s_{2}}\right)-W_{l}\left(g_{s_{1} l}^{C U}\right)$ is increasing in $\alpha_{l}$ and minimized at $f_{53}\left(1, \underline{\alpha}_{l}\right)>0$. $g_{s_{1} s_{2}} \succ_{l} g_{s_{1} l}$ given $f_{54}\left(\beta, \tau, \alpha_{l}\right)=$ $\left(W_{l}\left(g_{s l}\right)-W_{l}\left(g_{s_{1} s_{2}}\right)\right)+\beta\left(W_{l}\left(g_{l}^{H}\right)-W_{l}\left(g_{s l}\right)\right)+\beta^{2}\left(W_{l}\left(g^{F T}\right)-W_{l}\left(g_{l}^{H}\right)\right)$ is maximized at $f_{54}\left(\beta^{*}, 1, \underline{\alpha}_{l}\right)<0$ where $\beta^{*} \equiv \frac{1}{2} \frac{W_{l}\left(g_{l}^{H}\right)-W_{l}\left(g_{s l}\right)}{W_{l}\left(g_{l}^{H}\right)-W_{l}\left(g^{F T}\right)}$ solves $\frac{\partial f_{54}(\cdot)}{\partial \beta}=0$.

\section{Proof of Proposition 1}

In subgames at hub-spoke networks $g_{i}^{H}$, Lemma 1 implies $g_{i}^{H} \rightarrow g^{F T}$ arises unless $W_{l}\left(g_{s}^{H}\right)>W_{l}\left(g^{F T}\right)$ in which case $g_{s}^{H} \rightarrow g_{s}^{H}$ arises. In subgames at FTA insider-outsider networks $g_{i j}$, Lemma 2 and $\beta<\min \left\{\bar{\beta}^{F T-K}\left(\alpha_{l}, \tau\right), 1\right\}$ imply $g_{s_{1} s_{2}} \rightarrow g_{s_{1} s_{2}}$ arises if $g_{i j}=g_{s_{1} s_{2}}$ and $g_{s l} \rightarrow g_{l}^{H}$ arises if $g_{i j}=g_{s l}$. In subgames at CU insider-outsider networks $g_{i j}^{C U}$, Lemmas 3 together with $\tau<\bar{\tau}_{2}\left(\alpha_{l}\right)$ and $\alpha>\underline{\alpha}_{l}$ imply $g_{i j}^{C U} \rightarrow g_{i j}^{C U}$ arises. Before moving on, we note that $g_{s l} \succ_{s} g_{s_{1} s_{2}}^{C U}$ when $g_{s_{1} s_{2}}^{C U} \rightarrow g_{s_{1} s_{2}}^{C U}$ given $f_{11}\left(\beta, \tau, \alpha_{l}\right)=\left(W_{s}\left(g_{s l}\right)-W_{s}\left(g_{s_{1} s_{2}}^{C U}\right)\right)+$ $\beta\left(W_{s}\left(g_{l}^{H}\right)-W_{s}\left(g_{s l}\right)\right)+\beta^{2}\left(W_{s}\left(g^{F T}\right)-W_{s}\left(g_{l}^{H}\right)\right)$ is minimized at $f_{11}\left(\beta^{*}, 1, \underline{\alpha}_{l}\right)>0$ where $\beta^{*} \equiv \frac{1}{2} \frac{W_{s}\left(g_{s l}\right)-W_{s}\left(g_{l}^{H}\right)}{W_{s}\left(g^{F T}\right)-W_{s}\left(g_{l}^{H}\right)}$ solves $\frac{\partial f_{11}(\cdot)}{\partial \beta}=0$.

Now consider the subgame at the empty network $g=\varnothing$. In stage $1(\mathrm{c}), g_{s_{1} s_{2}}^{C U} \succ_{s_{2}} g_{s_{1} s_{2}} \succ_{s_{2}}$ $\varnothing$ by Lemma 4 . Thus, $s_{2}$ will accept a proposed CU or FTA from $l$ but it is optimal for $s_{1}$ to propose a CU. Hence, $\varnothing \rightarrow g_{s_{1} s_{2}}^{C U}$ arises in stage 1(c). For stages $1(\mathrm{~b})$ and $1(\mathrm{a})$, first suppose i) $\tau<\bar{\tau}_{1}\left(\alpha_{l}\right)$ or ii) $\tau \in\left(\bar{\tau}_{1}\left(\alpha_{l}\right), \bar{\tau}_{2}\left(\alpha_{l}\right)\right)$ and $\beta \notin\left(\underline{\beta}^{* \text { Flex }}\left(\alpha_{l}, \tau\right), \bar{\beta}^{* \text { Flex }}\left(\alpha_{l}, \tau\right)\right)$. In these cases, $g_{s_{1} s_{2}}^{C U} \succ_{l} g$ for $g \in\left\{g_{s l}, g_{s l}^{C U}, g^{F T}\right\}$ and thus it is optimal for $l$ to propose no agreement 
in stages 1(b) and 1(a). In turn, $\varnothing \rightarrow g_{s_{1} s_{2}}^{C U}$ arises and the equilibrium path of networks is $g_{s_{1} s_{2}}^{C U}$. Finally, suppose $\tau \in\left(\bar{\tau}_{1}\left(\alpha_{l}\right), \bar{\tau}_{2}\left(\alpha_{l}\right)\right)$ and $\beta \in\left(\underline{\beta}^{* \text { Flex }}\left(\alpha_{l}, \tau\right), \bar{\beta}^{* \text { Flex }}\left(\alpha_{l}, \tau\right)\right)$. In this case, given Lemma 4 (iii) and $g_{s l} \succ_{s} g_{s_{1} s_{2}}^{C U}$ from above, $s$ will accept an FTA from $l$ in stage 1(b) and, thus, $\varnothing \rightarrow g_{s l}$ arises because $g_{s l} \succ_{l} g_{s_{1} s_{2}}^{C U} \succ_{l} g_{s_{1} l}^{C U}$ implies it is optimal for $l$ to propose an FTA with $s$. By the same logic, $\varnothing \rightarrow g_{s l}$ also arises in stage 1(a). Thus the equilibrium path of networks is $\left(g_{s l}, g_{l}^{H}, g^{F T}\right)$.

Proof of Proposition 2

For subgames at hub-spoke networks and FTA insider-outsider networks, the logic follows the proof of Proposition 11. For subgames at CU insider-outsider networks, there is one difference from the proof of Proposition 1 and this difference arises when $\tau>\bar{\tau}_{3}\left(\alpha_{l}\right)$. Once $\tau>\bar{\tau}_{3}\left(\alpha_{l}\right), W_{i}\left(g^{F T}\right)>W_{i}\left(g_{s_{1} s_{2}}^{C U}\right)$ for $i=s_{1}, s_{2}, l$. Thus, in the subgame at $g_{s_{1} s_{2}}^{C U}$ it is optimal for $s_{1}$ and $s_{2}$ to accept a proposal of $g^{F T}$ from $l$ in stage 1 (a) and it is optimal for $l$ to propose $g^{F T}$. Thus, $g_{s_{1} s_{2}}^{C U} \rightarrow g^{F T}$ arises.

Now consider the subgame at the empty network $\varnothing$. Like the proof of Proposition 1 , $\varnothing \rightarrow g_{s_{1} s_{2}}^{C U}$ arises in stage 1(c). For stages 1(a) and 1(b) it is optimal for $s$ to accept an FTA or a CU from $l$ if $\beta<\bar{\beta}^{s}\left(\alpha_{l}, \tau\right)$ but it is only optimal to accept a CU (or $g^{F T}$ ) from $l$ if $\beta>\bar{\beta}^{s}\left(\alpha_{l}, \tau\right)$. Then, given $\beta \in\left(\underline{\beta}^{\text {Flex }}\left(\alpha_{l}, \tau\right), \bar{\beta}^{\text {Flex }}\left(\alpha_{l}, \tau\right)\right)$, it is optimal for $l$ to propose an FTA with a small country in stages $1(\mathrm{a})$ and $1(\mathrm{~b})$ when $\beta<\bar{\beta}^{s}\left(\alpha_{l}, \tau\right)$ but it is optimal for $l$ to offer a CU when $\beta>\bar{\beta}^{s}\left(\alpha_{l}, \tau\right)$. Thus, $\varnothing \rightarrow g_{s l}$ arises when $\beta<\bar{\beta}^{s}\left(\alpha_{l}, \tau\right)$ and but $\varnothing \rightarrow g_{s l}^{C U}$ arises when $\beta>\bar{\beta}^{s}\left(\alpha_{l}, \tau\right)$; the respective equilibrium path of networks is $\left(g_{s l}, g_{l}^{H}, g^{F T}\right)$ and $g_{s l}^{C U}$

\section{Proof of Proposition 3}

For subgames at hub-spoke networks as well as FTA and CU insider-outsider networks, the logic follows the proof of Proposition 2. Now consider the subgame at the empty network $\varnothing$. Like the proof of Proposition 2, $\varnothing \rightarrow g_{s_{1} s_{2}}^{C U}$ arises in stage 1(c). For stages 1(a) and 1(b) it is optimal for $s$ to accept a CU offer from $l$. Thus, given $\beta \notin\left(\underline{\beta}^{\text {Flex }}\left(\alpha_{l}, \tau\right), \bar{\beta}^{\text {Flex }}\left(\alpha_{l}, \tau\right)\right)$, it is optimal for $l$ to propose a $\mathrm{CU}$ with $s$ in stages $1(\mathrm{a})$ and $1(\mathrm{~b})$. Hence, $\varnothing \rightarrow g_{s l}^{C U}$ arises and the equilibrium path of networks is $g_{s l}^{C U}$

\section{Analysis when the large country's FT-K condition fails}

Now we consider the case where $\beta>\bar{\beta}_{l}^{F T-K}\left(\alpha_{l}, \tau\right)$. That is, conditional on FTA formation with $s, l$ refuses to become the hub despite knowing that $s$ will thus become the hub.

Proposition 5 characterizes the equilibrium. In doing so, we define $\bar{\beta}_{l}^{C U-K}\left(\alpha_{l}, \tau\right)$ as the 
threshold value of $\beta$ such that $\frac{1}{1-\beta} W_{l}\left(g_{s l}^{C U}\right)>W_{l}\left(g_{s l}\right)+\frac{\beta}{1-\beta} W_{l}\left(g_{s}^{H}\right)$, i.e. $g_{s l}^{C U} \succ_{l} g_{s l}$ in the subgame at the empty network, once $\beta>\bar{\beta}_{l}^{C U-K}\left(\alpha_{l}, \tau\right)$.

Proposition 5 Suppose $\beta>\bar{\beta}_{l}^{F T-K}\left(\alpha_{l}, \tau\right)$. If l prefers PTA formation with s over being a permanent $C U$ outsider, i.e. $g \succ_{l} g_{s_{1} s_{2}}^{C U}$ for some $g \in\left\{g_{s l}, g_{s l}^{C U}\right\}$ in the subgame at the empty network, then the equilibrium path of networks is $\left(g_{s l}, g_{s}^{H}\right)$ when $\beta<\bar{\beta}_{l}^{C U-K}\left(\alpha_{l}, \tau\right)$ but $g_{s l}^{C U}$ when $\beta>\bar{\beta}_{l}^{C U-K}\left(\alpha_{l}, \tau\right)$. Otherwise, the equilibrium path of networks is $g_{s_{1} s_{2}}^{C U}$.

Proof. For subgames at hub-spoke networks, the FTA insider-outsider network $g_{s_{1} s_{2}}$ and $\mathrm{CU}$ insider-outsider networks, the logic follows the proof of Proposition 2, However, $\beta>$ $\bar{\beta}_{l}^{F T-K}\left(\alpha_{l}, \tau\right)$ implies it is optimal for $l$ to propose no agreement in the subgame at $g_{s l}$ despite anticipation that $s_{1}$ offers an FTA to $s_{2}$, which is accepted, in stage $1(\mathrm{c})$. Thus, $g_{s l} \rightarrow g_{s}^{H}$ arises.

Now consider the subgame at the empty network $\varnothing$. As in Propositions 2 and $3, \varnothing \rightarrow g_{s_{1} s_{2}}^{C U}$ arises in stage 1(c). For stages 1(a) and 1(b) it is optimal for $s_{1}$ to accept an FTA or a CU from $l$ given $g \succ_{s} g^{\prime}$ for and $g \in\left\{g_{s_{1} l}, g_{s_{1} l}^{C U}\right\}$ and any $g^{\prime} \in\left\{g_{s_{2} l}, g_{s_{2} l}^{C U}, g_{s_{1} s_{2}}^{C U}\right\}$ given $W_{s}\left(g_{s l}\right)>$ $W_{s}\left(g_{s_{1} s_{2}}^{C U}\right)$ and $W_{s}\left(g_{s}^{H}\right)>W_{s}\left(g^{F T}\right)$. Thus, $l$ proposes no agreement when $g_{s_{1} s_{2}}^{C U} \succ_{l} g$ for $g \in\left\{g_{s l}, g_{s l}^{C U}\right\}$ and $\varnothing \rightarrow g_{s_{1} s_{2}}^{C U}$ arises with $g_{s_{1} s_{2}}^{C U}$ as the equilibrium path of networks. However, $l$ offers an FTA when $g_{s l} \succ_{l} g$ for $g \in\left\{g_{s_{1} s_{2}}^{C U}, g_{s l}^{C U}\right\}$ but offers a CU when $g_{s l}^{C U} \succ_{l} g$ for $g \in\left\{g_{s_{1} s_{2}}^{C U}, g_{s l}\right\}$. The respective transitions are $\varnothing \rightarrow g_{s l}$ and $\varnothing \rightarrow g_{s l}^{C U}$ with the respective equilibrium paths of networks $\left(g_{s l}, g_{s}^{H}\right)$ and $g_{s l}^{C U}$.

Unlike earlier propositions, a small country can emerge as the hub in the subgame at $g_{s l}$. However, no further liberalization occurs once this happens. Even though a small outsider country prefers to form an FTA with the large rather than the other small country, the large country refuses to become the hub when $\beta>\bar{\beta}_{l}^{F T-K}\left(\alpha_{l}, \tau\right)$. A necessary and sufficient condition for $\bar{\beta}_{l}^{F T-K}\left(\alpha_{l}, \tau\right)<1$ is $W_{l}\left(g_{s}^{H}\right)>W_{l}\left(g^{F T}\right)$. That is, when $l$ refuses FTA formation as a spoke it may also choose to forego temporary sole preferential access in both spoke markets as the hub in order to ensure global free trade is not attained. In such cases, a smaller country becomes the hub given it faces no threat of preference erosion.

The logic underlying the equilibrium structure closely follows that of earlier propositions. If PTA formation is not attractive enough to induce l's participation in liberalization when threatened with being a permanent CU outsider, the small countries form their own CU. This logic follows Proposition 1. Conversely, when PTA formation is attractive enough the logic follows that of Proposition 2 in that $l$ can effectively force $s$ to accept $l$ 's preferred PTA because $s$ cannot credibly threaten a CU with the other small country. Thus, the equilibrium path of agreements is $\left(g_{s l}, g_{s}^{H}\right)$ when $\beta<\bar{\beta}_{l}^{C U-K}\left(\alpha_{l}, \tau\right)$ but $g_{s l}^{C U}$ when $\beta>\bar{\beta}_{l}^{C U-K}\left(\alpha_{l}, \tau\right)$. 


\section{Analysis when large country is a close country}

\section{D.1 Welfare levels}

The notation follows Appendix A.

$$
W_{i}(g)=\sum_{z} C S_{i}^{z}(g)+\sum_{z} P S_{i}^{z}(g)+T R_{i}(g), i=s_{1}, s_{2}, l
$$

where

$$
\begin{aligned}
& \sum_{z} C S_{s_{1}}^{z}(g)=\frac{1}{2}\left[1-\tau\left(\frac{\tau^{2} t_{l s_{1}}^{g}+t_{l_{s_{2}}}^{g}+\alpha_{l}}{\left(2+\tau^{2}\right)}-t_{l_{s_{1}}}(g)\right)\right]^{2}+\frac{1}{2}\left[1-\tau \frac{\left.\tau^{2} t_{s_{2} s_{1}}^{g}+\alpha_{l}+t_{s_{2} l}^{g}\right)}{\left(2+\tau^{2}\right)}-t_{s_{2} s_{1}}^{g}\right]^{2} \\
& +\frac{1}{2}\left[1-\frac{\tau^{2}\left(t_{s_{1} l}^{g}+t_{s_{1} s_{2}}^{g}\right)+\tau\left(\alpha_{l}-1\right)+1}{\left(1+2 \tau^{2}\right)}\right]^{2} \\
& \sum_{z} C S_{s_{2}}^{z}(g)=\frac{1}{2}\left[1-\frac{\tau^{2} t_{l s_{1}}^{g}+t_{l s_{2}}^{g}+\alpha_{l}}{\left(2+\tau^{2}\right)}+t_{l s_{2}}(g)\right]^{2}+\frac{1}{2}\left[1-\frac{\left.\tau^{2} t_{s_{2} s_{1}}^{g}+\alpha_{l}+t_{s_{2} l}^{g}\right)}{\left(2+\tau^{2}\right)}\right]^{2} \\
& +\frac{1}{2}\left[1-\tau\left(\frac{\tau^{2}\left(t_{s_{1} l}^{g}+t_{s_{1} s_{2}}^{g}\right)+\tau\left(\alpha_{l}-1\right)+1}{\left(1+2 \tau^{2}\right)}-t_{s_{1} s_{2}}^{g}(g)\right)\right]^{2} \\
& \sum_{z} C S_{l}^{z}(g)=\frac{1}{2}\left[\alpha_{l}-\frac{\tau^{2} t_{l s_{1}}^{g}+t_{l_{s_{2}}}^{g}+\alpha_{l}}{\left(2+\tau^{2}\right)}\right]^{2}+\frac{1}{2}\left[\alpha_{l}-\frac{\tau^{2} t_{s_{2} s_{1}}^{g}+\alpha_{l}+t_{s_{2} l}^{g}}{\left(2+\tau^{2}\right)}+t_{s_{2} l}^{g}\right]^{2} \\
& +\frac{1}{2}\left[\alpha_{l}-\tau\left(\frac{\tau^{2}\left(t_{s_{1} l}^{g}+t_{s_{1} s_{2}}^{g}\right)+\tau\left(\alpha_{l}-1\right)+1}{\left(1+2 \tau^{2}\right)}-t_{s_{1} l}^{g}\right)\right]^{2} \\
& \sum_{z} P S_{s_{1}}^{z}(g)=\tau\left[\frac{t_{l s_{2}}^{g}+t_{s_{2 l}}^{g}+2 \alpha_{l}-2\left(t_{l s_{1}}^{g}+t_{s_{2} s_{1}}^{g}\right)}{\left(2+\tau^{2}\right)}\right] \\
& \sum_{z} P S_{s_{2}}^{z}(g)=\left[\frac{\alpha_{l}+\tau^{2}\left(t_{l_{1}}^{g}-t_{l_{s_{2}}}^{g}\right)-t_{l_{s_{2}}}^{g}}{\left(2+\tau^{2}\right)}+\tau\left[\frac{1+\tau\left(\alpha_{l}-1\right)+\tau^{2}\left(t_{s_{1} l}^{g}-t_{s_{1} s_{2}}^{g}\right)-t_{s_{1} s_{2}}^{g}}{\left(1+2 \tau^{2}\right)}\right]\right. \\
& \sum_{z} P S_{l}^{z}(g)=\left[\frac{\alpha_{l}+\tau^{2}\left(t_{s_{2} s_{1}}^{g}-t_{s_{2} l}^{g}\right)-t_{s_{2} l}^{g}}{\left(2+\tau^{2}\right)}+\tau\left[\frac{1+\tau\left(\alpha_{l}-1\right)+\tau^{2}\left(t_{s_{1} s_{2}}^{g}-t_{s_{1} l}^{g}\right)-t_{s_{1} l}^{g}}{\left(1+2 \tau^{2}\right)}\right]\right. \\
& T R_{s_{1}}(g)=t_{s_{1} s_{2}}^{g} \tau^{2}\left(\frac{\tau^{2}\left(t_{s_{1} l}^{g}+t_{s_{1} s_{2}}^{g}\right)+\tau\left(\alpha_{l}-1\right)+1}{\left(1+2 \tau^{2}\right)}-t_{s_{1} s_{2}}^{g}\right)+\tau t_{s_{1} l}^{g}\left[1-\alpha_{l}+\tau\left(\frac{\tau^{2}\left(t_{s_{1} l}^{g}+t_{s_{1} s_{2}}^{g}\right)+\tau\left(\alpha_{l}-1\right)+1}{\left(1+2 \tau^{2}\right)}-t_{s}^{g}\right.\right.
\end{aligned}
$$




$$
\begin{gathered}
T R_{s_{2}}(g)=t_{s_{2} s_{1}}^{g} \tau^{2}\left(\frac{\tau^{2} t_{s_{2} s_{1}}^{g}+t_{s_{2} l}^{g}+\alpha_{l}}{\left(2+\tau^{2}\right)}-t_{s_{2} s_{1}}^{g}\right)+t_{s_{2} l}^{g}\left[1-\alpha_{l}+\frac{\tau^{2} t_{s_{2} s_{1}}^{g}+t_{s_{2} l}^{g}+\alpha_{l}}{\left(2+\tau^{2}\right)}-t_{s_{2} l}^{g}\right] \\
T R_{l}(g)=t_{l s_{1}}^{g} \tau^{2}\left(\frac{\tau^{2} t_{l s_{1}}^{g}+t_{l s_{2}}^{g}+\alpha_{l}}{\left(2+\tau^{2}\right)}-t_{l s_{1}}^{g}\right)+t_{l s_{2}}^{g}\left[\frac{\tau^{2} t_{l s_{1}}^{g}+t_{l s_{2}}^{g}+\alpha_{l}}{\left(2+\tau^{2}\right)}-t_{l s_{2}}^{g}\right]
\end{gathered}
$$

Using the welfare equations reported above, optimal tariffs are easily derived (as in the main text) and used to obtain the formulae for welfare levels under all possible networks.

\section{D.2 Proof of Proposition 4}

The proof proceeds by construction. As stated in the text, we suppose $W_{s}\left(g^{F T}\right)>W_{s}\left(g_{l}^{H}\right)>$ $W_{s}\left(g_{s^{\prime} l}\right)$ for $s^{\prime} \neq s$. Moreover, we suppose $W_{s}\left(g^{F T}\right)>W_{s}\left(g_{s_{1} s_{2}}^{C U}\right)$. Together, these two conditions imply a lower bound on $\alpha_{l}$ and $\tau$, denoted $\underline{\alpha}_{l}$ and $\underline{\tau}$. In addition, define $\bar{\alpha}_{l}$ as the threshold value of $\alpha_{l}$ such that $W_{l}\left(g_{s_{1} l}\right)+\beta W_{l}\left(g_{l}^{H}\right)+\frac{\beta^{2}}{1-\beta} W_{l}\left(g^{F T}\right)>\frac{1}{1-\beta} W_{l}\left(g_{s_{1} s_{2}}^{C U}\right)$ for all $\beta \in[0,1)$ and for some $\tau>\underline{\tau}$ when $\alpha_{l}<\bar{\alpha}_{l} \cdot{ }^{29}$ We condition the subsequent analysis on $\tau>\underline{\tau}$ and $\alpha_{l} \in\left(\underline{\alpha}_{l}, \bar{\alpha}_{l}\right)$.

For subgames at hub-spoke networks $g_{i}^{H}$, Lemma 1 still applies given $\tau>\underline{\tau}$ and $\alpha_{l} \in$ $\left(\underline{\alpha}_{l}, \bar{\alpha}_{l}\right)$. Thus, $g_{i}^{H} \rightarrow g^{F T}$ arises unless $i=s$ and $W_{l}\left(g^{F T}\right)>W_{l}\left(g_{s}^{H}\right)$ in which case $g_{i}^{H} \rightarrow g_{i}^{H}$.

For subgames at FTA insider-outsider networks $g_{i j}$, Lemma 2 still implies $g_{s_{1} s_{2}} \rightarrow g_{s_{1} s_{2}}$ given $W_{l}\left(g_{s_{1} s_{2}}\right)>\max \left\{W_{l}\left(g^{F T}\right), W_{l}\left(g_{s}^{H}\right)\right\}$ when $\tau>\underline{\tau}$ and $\alpha_{l} \in\left(\underline{\alpha}_{l}, \bar{\alpha}_{l}\right)$. For subgames at $g_{s l}$, as in Lemma 2, $g_{s l} \rightarrow g_{s}^{H}$ arises if $\beta>\bar{\beta}_{l, s}^{F T-K}\left(\alpha_{l}, \tau\right)$ while $g_{s l} \rightarrow g_{l}^{H}$ arises if $\beta<\bar{\beta}_{l, s}^{F T-K}\left(\alpha_{l}, \tau\right)$ and $\beta<\max \left\{\hat{\beta}_{l, s}\left(\alpha_{l}, \tau\right), \hat{\beta}_{s}\left(\alpha_{l}, \tau\right)\right\} .{ }^{30}$ But, unlike in Lemma 2 , we can now have $\beta \in\left(\max \left\{\hat{\beta}_{l, s}\left(\alpha_{l}, \tau\right), \hat{\beta}_{s}\left(\alpha_{l}, \tau\right)\right\}, \bar{\beta}_{l, s}^{F T-K}\left(\alpha_{l}, \tau\right)\right)$. In this case, remaining a permanent insider is most preferred for $s$ and $l$. Thus, it is optimal for $l$ to propose no agreement in stage 1(a) since no agreement will emerge in stage 1(c) given it is optimal $s_{1}$ to propose no agreement if $g=g_{s_{1} l}$ and optimal for $s_{2}$ to refuse an agreement if $g=g_{s_{2} l}$. Hence, $g_{s l} \rightarrow g_{s l}$ arises when $\beta \in\left(\max \left\{\hat{\beta}_{l, s}\left(\alpha_{l}, \tau\right), \hat{\beta}_{s}\left(\alpha_{l}, \tau\right)\right\}, \bar{\beta}_{l, s}^{F T-K}\left(\alpha_{l}, \tau\right)\right)$.

For subgames at CU insider-outsider networks $g_{i j}^{C U}$, the proof of Lemma 3 makes it clear that $g_{i j}^{C U} \rightarrow g^{F T}$ iff $W_{i}\left(g^{F T}\right)>W_{i}\left(g_{i j}^{C U}\right)$ for $i=s_{1}, s_{2}, l$. Given $\tau>\underline{\tau}$ and $\alpha_{l}>\underline{\alpha}_{l}$, $g_{s_{1} s_{2}} \rightarrow g^{F T}$ iff $W_{l}\left(g^{F T}\right)>W_{l}\left(g_{s_{1} s_{2}}^{C U}\right)$ and $g_{s_{1} s_{2}}^{C U} \rightarrow g_{s_{1} s_{2}}^{C U}$ iff $W_{l}\left(g^{F T}\right)<W_{l}\left(g_{s_{1} s_{2}}^{C U}\right)$. Moreover, $g_{s_{1} l} \rightarrow g_{s_{1} l}$ given $W_{s_{1}}\left(g_{s_{1} l}^{C U}\right)>W_{s_{1}}\left(g^{F T}\right)$. Whether $g_{s_{2} l}^{C U} \rightarrow g^{F T}$ or $g_{s_{2} l}^{C U} \rightarrow g_{s_{2} l}^{C U}$ is irrelevant for the remainder of the proof.

${ }^{29}$ Numerically, the critical values correspond to $\underline{\tau} \approx .68, \underline{\alpha}_{l} \approx 1.11$ and $\bar{\alpha}_{l} \approx 1.165$.

${ }_{30} \bar{\beta}_{l}^{F T-K}\left(\alpha_{l}, \tau\right)$ and $\hat{\beta}_{l}\left(\alpha_{l}, \tau\right)$ now have $s$ subscripts since the small countries are now asymmetric. 
Finally, consider the subgame at the empty network. In stage $1(\mathrm{c})$, we have $W_{s_{1}}\left(g_{s_{1} s_{2}}^{C U}\right)>$ $\max \left\{W_{s_{1}}\left(g_{s_{1} s_{2}}\right), W_{s_{1}}(\varnothing)\right\}$ and $W_{s_{2}}\left(g_{s_{1} s_{2}}^{C U}\right)>W_{s_{2}}(\varnothing)$ so $s_{2}$ will accept a CU offer from $s_{1}$ and it is optimal for $s_{1}$ to offer a CU to $s_{2}$. Thus, $\varnothing \rightarrow g_{s_{1} s_{2}}^{C U}$ arises in stage $1(\mathrm{c})$.

For stages $1(\mathrm{a})$ and $1(\mathrm{~b})$, suppose that $g_{s_{1} l} \rightarrow g_{l}^{H} \rightarrow g^{F T}$. Then, given $\hat{\beta}_{s_{2}}\left(\alpha_{l}, \tau\right)>$ $\hat{\beta}_{s_{1}}\left(\alpha_{l}, \tau\right)$ and $\bar{\beta}_{l, s_{1}}^{F T-K}\left(\alpha_{l}, \tau\right)<\bar{\beta}_{l, s_{2}}^{F T-K}\left(\alpha_{l}, \tau\right), g_{s_{2} l} \rightarrow g_{l}^{H} \rightarrow g^{F T}$. Thus, it is optimal for $s_{1}$ and $s_{2}$ to accept an offer of $g^{F T}$ in stage $1\left(\right.$ a) because $g^{F T} \succ_{s} g$ for any $g \in\left\{g_{s_{1} s_{2}}^{C U}, g_{s^{\prime} l}^{C U}, g_{s^{\prime} l}\right\}$ where $s^{\prime} \neq s$ given $W_{s}\left(g^{F T}\right)>W_{s}\left(g_{s^{\prime} l}\right)$. Moreover, it is optimal for $s_{1}$ to accept an FTA offer from $l$ in stage 1 (a) if $g_{s_{1} l} \succ_{s_{1}} g$ for any $g \in\left\{g_{s_{1} s_{2}}^{C U}, g_{s_{2} l}^{C U}, g_{s_{2} l}\right\}$. Indeed, given three observations, a sufficient condition for this to hold is $g_{s_{1} l} \succ_{s_{1}} g_{s_{1} s_{2}}^{C U}$ where $g_{s_{1} s_{2}}^{C U} \rightarrow g^{F T}$; this reduces to $\tau<\hat{\tau}_{1}\left(\alpha_{l}\right)$. These three observations are (a) $W_{s_{1}}\left(g_{s_{1} l}\right)>W_{s_{1}}\left(g_{s_{2} l}\right)$, (b) $W_{s_{1}}\left(g^{F T}\right)>W_{s_{1}}\left(g_{s_{1} s_{2}}^{C U}\right)>W_{s_{1}}\left(g_{s_{2} l}^{C U}\right)$, and $(\mathrm{c}) V_{s_{1}}\left(\left(g_{s_{1} l}, \ldots\right)\right)>V_{s_{1}}\left(\left(g_{s_{1} s_{2}}^{C U}, g^{F T}\right)\right)$ for all $\tau$ when $g_{s_{1} s_{2}}^{C U} \rightarrow g_{s_{1} s_{2}}^{C U}$.

In determining $l$ 's optimal action, we define the following critical values: i) $W_{l}\left(g_{s_{1} l}\right)>$ $W_{l}\left(g_{s_{1} l}^{C U}\right)$ iff $\tau<\hat{\tau}_{2}\left(\alpha_{l}\right)$ and ii) $W_{l}\left(g^{F T}\right)>W_{l}\left(g_{s_{1} l}^{C U}\right)$ iff $\tau<\hat{\tau}_{3}\left(\alpha_{l}\right)$. Then, given two observations, we have $g_{s_{1} l} \succ_{l} g$ for any $g \in\left\{g_{s_{1} l}^{C U}, g_{s_{2} l}^{C U}, g_{s_{2} l}\right\}$ and for all $\beta \in[0,1)$ when $\tau<$ $\min \left\{\hat{\tau}_{1}\left(\alpha_{l}\right), \hat{\tau}_{2}\left(\alpha_{l}\right)\right\}$. These two observations are (a) $W_{l}\left(g_{l}^{H}\right)>\max \left\{W_{l}\left(g^{F T}\right), W_{l}\left(g_{s_{1} l}^{C U}\right), W_{l}\left(g_{s_{2} l}^{C U}\right)\right\}$ and (b) $W_{l}\left(g_{s_{1} l}\right)>W_{l}\left(g_{s_{2} l}\right)$. Nevertheless, we actually have $g_{s l} \succ_{l} g^{F T}$ iff $W_{l}\left(g_{s l}\right)+$ $\beta W_{l}\left(g_{l}^{H}\right)+\frac{\beta^{2}}{1-\beta} W_{l}\left(g^{F T}\right)>\frac{1}{1-\beta} W_{l}\left(g^{F T}\right)$ which reduces to $\beta>\frac{W_{l}\left(g^{F T}\right)-W_{l}\left(g_{s l}\right)}{W_{l}\left(g_{l}^{H}\right)-W_{l}\left(g^{F T}\right)} \equiv \tilde{\beta}_{l, s}\left(\alpha_{l}, \tau\right)$. Thus, it is optimal for $l$ to offer $g^{F T}$, which is then accepted, if $\beta<\tilde{\beta}_{l, s_{1}}\left(\alpha_{l}, \tau\right)$. In this case, $\varnothing \rightarrow g^{F T}$ arises and the equilibrium path of networks is $g^{F T}$. However, once $\beta>\tilde{\beta}_{l, s_{1}}\left(\alpha_{l}, \tau\right)$, it is optimal for $l$ to offer an FTA to $s_{1}$ iff $g_{s_{1} l} \succ_{l} g_{s_{1} s_{2}}^{C U}$ which reduces to $\tau<\hat{\tau}_{4}\left(\alpha_{l}\right)$. Therefore, letting $\bar{\tau}^{\prime}\left(\alpha_{l}\right) \equiv \min \left\{\hat{\tau}_{1}\left(\alpha_{l}\right), \hat{\tau}_{2}\left(\alpha_{l}\right), \hat{\tau}_{3}\left(\alpha_{l}\right), \hat{\tau}_{4}\left(\alpha_{l}\right)\right\}$, it is optimal for $s_{1}$ to accept an FTA offer from $l$ and it is optimal for $l$ to offer an FTA to $s_{1}$ in stage 1 (a) if $\tau<\bar{\tau}^{\prime}\left(\alpha_{l}\right)$. Thus, conditional on $g_{s_{1} l} \rightarrow g_{l}^{H} \rightarrow g^{F T}$, the equilibrium path of networks is $\left(g_{s_{1} l}, g_{l}^{H}, g^{F T}\right)$ when $\tau<\bar{\tau}^{\prime}\left(\alpha_{l}\right)$ and $\beta>\tilde{\beta}_{l, s_{1}}\left(\alpha_{l}, \tau\right)$.

As discussed above, $g_{s l} \rightarrow g_{l}^{H}$ need not arise in the subgame at $g_{s l}$. Rather, $g_{s l} \rightarrow g_{s}^{H}$ or $g_{s l} \rightarrow g_{s l}$ could arise; and, if so, $\tilde{\beta}_{l, s_{1}}\left(\alpha_{l}, \tau\right)>1$. Moreover, these alternative network paths increase the continuation payoff for $s$ and $l$ but decrease the continuation payoff for $s^{\prime} \neq s$. Thus, given $\hat{\beta}_{s_{2}}\left(\alpha_{l}, \tau\right)>\hat{\beta}_{s_{1}}\left(\alpha_{l}, \tau\right)$ and $\bar{\beta}_{l, s_{1}}^{F T-K}\left(\alpha_{l}, \tau\right)<\bar{\beta}_{l, s_{2}}^{F T-K}\left(\alpha_{l}, \tau\right)$, the same logic as above implies it is optimal for $l$ to offer an FTA to $s_{1}$ in stage 1(a) and it is optimal for $s_{1}$ to accept. Thus, in this case, the equilibrium path of networks is either $\left(g_{s_{1} l}, g_{s}^{H}\right)$ or $g_{s_{1} l}$.

\section{References}

Aghion, P., Antrās, P., Helpman, E., 2007. Negotiating free trade. Journal of International Economics 73 (1), 1-30. 
Appelbaum, E., Melatos, M., 2013. How does uncertainty affect the choice of trade agreements?

Bagwell, K., Staiger, R., 1997. Multilateral tariff cooperation during the formation of Customs Unions. Journal of International Economics 42 (1), 91-123.

Bagwell, K., Staiger, R., 1999. Regionalism and multilateral tariff cooperation. In: Pigott, J., Woodland, A. (Eds.), International trade policy and the Pacific rim. Macmillan.

Bagwell, K., Staiger, R. W., 2010. Backward stealing and forward manipulation in the WTO. Journal of International Economics 82 (1), 49-62.

Baier, S. L., Bergstrand, J. H., Clance, M. W., 2014. Preliminary examination of heterogeneous effects of Free Trade Agreements. Mimeo.

Baier, S. L., Bergstrand, J. H., 2004. Economic determinants of free trade agreements. Journal of International Economics 64 (1), 29-63.

Bond, E. W., Riezman, R. G., Syropoulos, C., 2004. A strategic and welfare theoretic analysis of Free Trade Areas. Journal of International Economics 64 (1), 1-27.

Broda, C., Limao, N., Weinstein, D. E., 2008. Optimal tariffs and market power: the evidence. The American Economic Review 98 (5), 2032-2065.

Chen, M., Joshi, S., 2010. Third-country effects on the formation of Free Trade Agreements. Journal of International Economics 82 (2), 238-248.

Egger, P., Larch, M., 2008. Interdependent preferential trade agreement memberships: An empirical analysis. Journal of International Economics 76 (2), 384-399.

Eichengreen, B., Irwin, D., 1998. The role of history in trade flows. In: M. Canzoneri, W. J. E., Grilli, V. (Eds.), The Regionalization of the World Economy. University of Chicago Press.

Estevadeordal, A., Freund, C., Ornelas, E., 2008. Does regionalism affect trade liberalization toward nonmembers? The Quarterly Journal of Economics 123 (4), 1531-1575.

Facchini, G., Silva, P., Willmann, G., 2012. The Customs Union issue: Why do we observe so few of them? Journal of International Economics 90, 136-147.

Feenstra, R. C., 2004. Advanced international trade: theory and evidence. Princeton University Press. 
Freund, C., McLaren, J., 1999. On the dynamics of trade diversion: Evidence from four trade blocks. Board of Governors of the Federal Reserve System, International Finance Discussion Paper No. 637.

Furusawa, T., Konishi, H., 2007. Free trade networks. Journal of International Economics $72(2), 310-335$.

Gatsios, K., Karp, L., 1991. Delegation games in customs unions. The Review of Economic Studies 58 (2), 391-397.

Jackson, M., 2008. Social and economic networks. Princeton University Press.

Lake, J., 2014. Why don't more countries form Customs Unions instead of Free Trade Agreements? The role of flexibility. Mimeo.

Ludema, R., 1991. International trade bargaining and the Most-Favored-Nation clause. Economics \& Politics 3 (1), 1-20.

Ludema, R. D., 2002. Increasing returns, multinationals and geography of preferential trade agreements. Journal of International Economics 56 (2), 329-358.

McLaren, J., 2002. A theory of insidious regionalism. Quarterly Journal of Economics 117 (2), $571-608$.

Melatos, M., Dunn, S., 2013. Flexibility in trade bloc design. Mimeo.

Melatos, M., Woodland, A., 2007. Endogenous trade bloc formation in an asymmetric world. European Economic Review 51 (4), 901-924.

Missios, P., Saggi, K., Yildiz, H., 2014. External trade diversion, exclusion incentives and the nature of preferential trade agreements. Mimeo.

Ornelas, E., 2008. Feasible multilateralism and the effects of regionalism. Journal of International Economics 74 (1), 202-224.

Ornelas, E., Liu, X., 2012. Free Trade Agreements and the consolidation of democracy. Mimeo.

Ray, D., Vohra, R., 1997. Equilibrium binding agreements. Journal of Economic Theory $73(1), 30-78$.

Richardson, M., 1993. Endogenous protection and trade diversion. Journal of International Economics 34 (3), 309-324. 
Riezman, R., 1999. Can bilateral trade agreements help to induce free trade? Canadian Journal of Economics 32 (3), 751-766.

Roberts, M., Tybout, J., 1997. The decision to export in Colombia: an empirical model of entry with sunk costs. American Economic Review 87 (4), 545-564.

Saggi, K., Woodland, A., Yildiz, H. M., 2013. On the relationship between preferential and multilateral trade liberalization: the case of Customs Unions. American Economic Journal: Microeconomics 5 (1), 63-99.

Saggi, K., Yildiz, H., 2010. Bilateralism, multilateralism, and the quest for global free trade. Journal of International Economics 81 (1), 26-37.

Saggi, K., Yildiz, H. M., 2009. Optimal tariffs of preferential trade agreements and the tariff complementarity effect. Indian Growth and Development Review 2 (1), 5-17.

Seidmann, D., 2009. Preferential trading arrangements as strategic positioning. Journal of International Economics 79, 143-159.

Soegaard, C., 2013. An oligopolistic theory of regional trade agreements. Tech. rep.

Syropoulos, C., 2003. Rules for the disposition of tariff revenues and the determination of common external tariffs in Customs Unions. Journal of International Economics 60 (2), $387-416$.

WTO, 2011. World Trade Report 2011. The WTO and preferential trade agreements: From co-existence to coherence.

Zissimos, B., 2011. Why are trade agreements regional? Review of International Economics $19(1), 32-45$. 